Vittheilurgen aus dem Königlichen Vaturalien-Cabinet

$$
\text { with croupliments }
$$
in Stuttgart No. $2 \%$.

NEUE ZEUGLODONTEN AUS DEM UNTEREN MITTELEOCÄN VOM MOKATTAM BEI CAIRO.

VON

PROF. D. E. FRAAS.

MIT 3 TAFELN.

J E N A,

VERLAG VON GUSTAV FISCIIER.

1904. 

Mittheilungen aus dem Königlichen Naturalien-Cabinet in Stuttgart No. 27.

\section{NEUE ZEUGLODONTEN AUS DEM UNTEREN MITTELEOCÄN VOM MOKATTAM BEI CAIRO.}

VON

PROF. DR. E. FRAAS.

MIT 3 TAFELN.

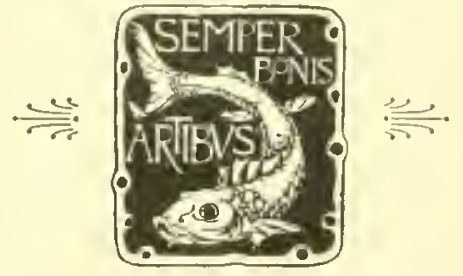

J E N A,

VERLAG VON GUSTAV FISCHER.

1904. 
Uebersetzungsrecht vorbehalten. 


\title{
Neue Zeuglodonten aus dem unteren Mitteleocän vom Mokattam bei Cairo.
}

\author{
Von
}

\author{
Prof. Dr. E. Fraas.
}

Die Kenntniss der Zenglodonten, der bisherigen einzigen Vertreter der Urwale oder Archäoceten, ist in den letzten Jahren durch einen hervorragenden Fumd erweitert worden, welchen E. v. Stromer in dem oberen Mitteleocän des $\mathrm{F}$ aj îm gemacht hat, und worüber er verschiedenfach berichtet hat ${ }^{1}$ ). Es handelte sich um einen gut erhaltenen Schädel sammt Unterkiefer von Zenglodon Osiris Dames und um einige nnbedentendere, aber paläontologisch selı wichtige Ueberreste einer kleineren Art, die Stroner als Zouglodon Zitteli beschrieh. Die Fundstücke stammen, wie die seinerzeit vou Schweinfurth gesammelten und von Danes²) beschriebenen Reste des Zenglodon Osiris aus dem Fajûm und rrurden von Stromer und MI. BLANCKENHORN gelegentlich einer im Winter 1901 auf 1902 von der bayrischen Akademie der Wissenschaften veranlassten Studienreise aufgefunden, wobei zugleich genane Aufnahmen über die geologischen Horizonte der ron BLANCKENHORN als „Zeuglodonberg" beschriebenen Fundstätte 3 Stunten west-südwestlich von Qasp-es-Saga gemacht wurden ${ }^{3}$.

E. v. Stroner nimmt bei der Bearbeitung seines Materiales Gelegenheit, nicht allein die aus seinem Funde sich ergebenlen Ergänzungen im Schädel- und Skeletbau der Zenglodonten zı bespreclıen, sondern er geht anch unter Beiziehnng aller bis jetzt beschriebenen Ueberreste auf die allgemeinen Fragen über den Bau des Skelettes, die Lebensweise und systematische Stellung dieser eigenartigen Walthiere ein, und seine klare und erschöpfende Darstellung enthebt mich der mühevollen Arbeit, welche

1) E. STroner, Ein Schüdel und Unterkiefer von Zeuglodon Osiris DAmes. Sitzungsber. d. math.-phys. Cl. d. Kgl. bayr. Akad. d. Wiss. Bd. 32. München 1902, pag. 345. - Ders., Zenglodon-Reste aus dem obcren Mitteleocän des F a j t m. Beiträge z. Paläontolog. n. Geol. Oesterreich-Ungarns und des Orients. Bd. 20. Heft 2 u. 3. Wien 1903. Ders., Einiges über Bau und Stellung der Zenglodonten. Monatsber. d. deutsch. geolog. Ges. 1903. No. 2.

2) W. Danes, Ueber Zeuglodonten aus A egypten und die Bezichungen der Archäoceten zn den übrigen Cetaceen. Paläontolog. Abhandlg. von DAMEs nnd KAYser. N. F. I. Heft 5. 1894.

3) M. Blanckevions, Nene geologisch-stratigraphische Beobachtungen in A egypte u. Sitznngsber. der math.phys. Cl. d. Kgl. bayr. Akad. d. INiss. Bd. 32. 190?. Heft 3, pag. 390. 
insbesondere die Durcharbeitung der illeren, vielfach unklaren Literatur verursacht. Ebenso enthebt sie mich einer erneuten Znsammenstellung der freilich nicht sehr ansgedehnten Literatur, da diese sich bei Stromer (Beiträge z. Paläont. u. Geol. Oesterreich-Ungarns etc., l. c. pag. 100) bereits vorfindet.

In eben dieser Arbeit (pag. 83-85) heschreibt E. Stroner die durch gewaltige Grösse nnd

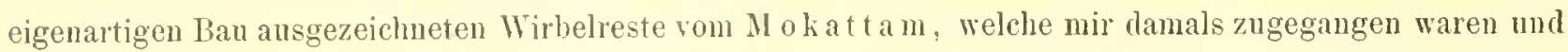
die ich ihm noch in letzter Stunde zm Bearbeitung übergeben konnte. Mit Recht weist er darauf hin, dass es sich dabei mm eine für Aegypten nene grosse Art hanlelt, welche Uebereinstimmung mit lem amerikanischen Zeuglodon macrospondylus: zeigt.

In dem letzten Jahre gingen an das Kgl. Natmalien-Cabinet in Stuttgart eine Reihe nener Fundstiicke ron Zeuglorlonten ans dem $\mathrm{M}$ ok a $\mathrm{t}$ a $\mathrm{m}$ bei $\mathrm{C}$ a i r o ein, welche geeignet sind, unsere Kienntniss noch wesentlich zn erweitern, und deshall, eine Bearbeitung beanspruchen. Ich verdanke diese wichtigen Funde neben einer grossen Collection anderer Ueberreste meinem unermüdlichen Sammler MarkGRAF in Cairo, der seinerseits unterstïtzt wurle durch zwei Gönner unserer Sammlung, Herrn G. Mez in Cairo und Herrn T'n. Wanner in Stuttgart. die Ilerrn Markgraf es emöglicht haben, mehrere Jahre hindurch eingehende und rom hesten Erfolg gekrönte Anfsammlungen im Mokattam zu machen. Ausser Zeuglodon umfassen die Aufsammlnngen inshesondere die eocänen Sirenen, Eotherimm und Protosiren, über welche in Bälde O. ABEL in Wien berichten wird, einen hübschen, an Tomistoma Eygenburgensis Toula erimnernden Crocodilier, zahlreiche Fischreste, die E. STromer bearbeitet, Brachiuren von schöner Erhaltung mul grossem Formenreichthum, welche J. LOERENTHEY sich zm Bearbeitung vorbehalten hat, und natüllich eine Menge von Invertebraten mu Früchte von Apeibopsis und anderen Arten. Es ist eine hillige Pflicht, den drei llerren Markgraf, Mez mil Wanner den gebührenden Dank auch an dieser Stelle anszusprechen, denn ilnen verdankt nicht nur unser Museum ein überaus reiches Material, sondern anch die paläontologische Wissenschaft als solche eine entschiedene Bereichermng.

\section{Geologischer Horizont der Funde.}

Herru E. Stromer, der sich z. Z. in A gypten befindet, um dort erneute Aufsammlungen zu machen. rerdanke ich in einer brieflichen Mittheilung eine genane Feststelhng der geologisehen Horizonte, ans welchen meine Fnndstïcke stammen. und ich spreche ihn hierfür meinen besten Dank ans. Die l'rofile am Mokattam sind schon vielfach Gegenstand eingehender Bearbeitung gewesen ') nnd dürfen nach den letzten Untersuchungen von BLANCKENHORN vorlänfig als geklärt nul abgeschlossen betrachtet werden. Der Horizont der als Irotocetus atarns zu beschreibenden Ueberreste konnte von E. Stroner genau bestinmt werden und fält zusammen mit der Schicht A 1 e Schweinfurtis's, die ihrerseits mit dem Horizont I a Mayer-Eymar's ident ist md vom Blanckenhon an die Basis des Gizehensis-Lagers $I_{2}$ gestellt wird. Jedenfalls scheint es sich um das tiefste am Mokattam auftretende Schichtenglied zu handelı. Aus der tiefsten Lage stammt ein grosser Krnochenfisch, der zu den Leryciden gehört, ein Schädel von Arins, sowie einige Selachierwirbel. $3 \mathrm{~m}$ darïber ist das Lager von Protoctus atums vergesellschaltet mit zahlreichen Krabben ans den Familien Palacocarpitius,

1) Ausser vielen Anderen vergl. G. Schwenfunth, Ueber die geologische sichichtenglicderung des Moka $t \mathrm{tam}$ bei Cairo. Zeitschr. d. deutseh. geol. Ges. Bd. 35. 1883, pag. 709. - M. BLAnchenhors, Neues zur Geologie und Paläontologie Aegyptens. II. l)as Paläogen. Zeitschr. d. deutsch. geol. Ges. Bul. 55. 1900, pag. 427. - Derselbe, Sitzungsber. d. math. phys. Cl. d. Kggl. bayr. Akarl. d. Wiss. 13d. 32. 1902. Heft 3, pag. 364. 
Titanorncimus, Micromaja, Loborarmins u. a., sowie den Resten der von Areu als Protosiren bezeichneten Arten. Etwa $4 \mathrm{~m}$ darüher lagert eine Kalkbank ans welcher Reste von Tomistoma n. s]. und Eotherimm acyyptiacum Owen stammen, und in den weiterhin folgenlen $10 \mathrm{~m}$ wurden hauptsäehlich Zähne von Haifischen, Myliobatiten, Ancistrodon, Liodom. Plethodus und veremzelte Sirenen-Reste gefunden.

Diese ganze Sehichtenserie fällt zusammen mit Blaxckeniorss ,Gizehensis-Lager", während die Reste des grossen Mesocetus Srmumfurthi aus emer bedentend höheren Schicht stammen. Sie lagen naeh E. Stromer etwa 8 m unter der Obergrenze des unteren Mokattan in einer harten grauweissen Kalkbank, mnter weleher Schivaster sehr häufig ist. Diese ist erfïlt nit Conchylienresten und voll relativ kleiner Nummuliten, und entspricht der Oberregion von A 1 a Schweinfurtu's (1. C. S. 730), wïlnenl sie Buanckenhors als "zweite Maner" I, 5 resp. als oberstes Glied der unteren II okatt amst $u$ fe bezeicluet.

Es unterliegt demnach keinem Zweifel. dass beide Zeuglodontenfunde, welche ich später zu beschreiben habe, aus der unteren Mokattamstufe, d. h. dem unteren Mitteleocän, stammen und sie unterseheiden sich demnach schon durch ihr geologisches Alter ron den frïheren aus A g g pten beschriebenen Funden, welche nach den Untersuchungen von Stromer und BLANckenHors in die Horizonte II 1 und II, 5 a der oberen Mokattamstnfe zn stellen sind. Die Reste aus dem Horizonte II 1 gehören einer nit Mesocetus Soluceinfurthi identen oder nahe verwandten Art an.

Ans dem höheren Horizoute II $5 \varkappa$ stammen die von Danes, Stroner, E. Smith ${ }^{1}$ ) und C. W. ANDREws ${ }^{2}$ ) erwähnten Reste von Zemglorlon Osiris, Z. Zitteli und einer etwaigen nenen Art, von welcher E. Sмiтн den Gehirnansguss beschrieben hat.

\section{Beschreibung der Arten. \\ Protocetus atavus n. g., n. sp.}

Die Funde, welehe im Frïljalı 190; von Herm Markgraf in dem unteren Gizehensis-Lager gemacht wurleu, umfassen zunäehst eine grössere Anzahl zusammengehöriger Skelettheile, welche in grossen zusammenpassenden Kalksteinstïcken eingeschlossen. in meine Hand kamen, so dass an der Zusammengehörigkeit zu einem einzigen Indivilumm nicht zu zweifeln ist. Die Präparation war theilweise eine recht schwierige und beanspruchte grosse Aufmerksamkeit, da sich rlie lichtgefärbten Krnoehen kaum vou dem ungebenden Gesteine abhoben. Da Schädel, Wirbel und Rippen ziemlich regellos durcheinandergedrängt lagen und sich durchkrenzten, so war es leider trotz aller Sorgfalt nicht zu vermeiden, dass einzelne Knoehenreste zertrimmert und abgesprengt werden mussten, un anlere besser erhaltene hlosszulegen. Tach Möglichkeit wurden alle Skeletstïcke vollständig frei herausgearbeitet, was natürlich bei den Wirbeln mit ihren Fortsätzen besondere Schwierigkeiten bereitete, aber doch für die Bearbeitung dringend erforterlich erschien.

Der Erhaltungszustand kann im grossen Ganzen als ein vorzïglicher bezeiclınet werden und wäre noch viel besser, wenn die Kalkblöcke nicht im Steinbruch von den Arbeitern zertriimmert worden wären, wobei vielfach der Knochen verletzt wurde und eiuzelne Bruchstücke verloren gegangen sind. Um den Stiicken Halt zu verleihen, mnssten die fehlenden Stellen mit lïttmasse ausgefüllt werden,

1) G. Elliot Sinth, The Brain of the Archaeoceti, Proc. Royal Soc. Vol. 71. London 1903, pag. 322.

2) C. W. Axdrews, Extinct Vertebrates from Egypt, Geol. Magazine, N. S. Decade IV, Vol. S, 1901, pag. 437; ibid., September 1901, pag. 401. 
jedoch handelt es sich dahei nie um eigentliche Ergänzungen, durch welche etwaige Unsicherheiten entstehen könnten. Anf den Abbildungen ist the Kittmasse dureh Schraftur angregeben. Sehr vortheilhaft ist, dass das Schädelstïck und die vordere Hälfte der Wirbelsäule ganz frei von Verdrücknng und Deformirung war und sich die Oherthäche des Kinochens gut aus lem umgebenten Káalksteine heransschälte. Die grösste Schwierigkeit hereiteten die Zïhne, bei welchen zwar der Schmelz selnr schïn erhalten. aber ebenso wie das Dentin von einer Brïchigkeit war, welche häufig der sorgfältigsten Präparation Holn sprach. Immerhin gelang es auch rom Gehiss eiu klares lild zu bekonmen. Der Erhaltungszustand wurde in der hinteren Mälfte der Wirbelsäule schlechter, inden hier die Wirbel vielfach verdrïckt waren und sich del Knochen kaum rom umgebenden (iesteine ablöste. Ein Gesteinsstiock, in welchem offenbar die Reste von Schwanzwirbeln eingebettet waren, widerstand allen Bemïhnngen und es erwies sich schliesslich als unmöglich, diese Skelettheile herauszupräpariren.

Eine Ergänzung bildet eine Seric von 5 Wirbeln ans der hinteren Rumpfregion. welche einer späteren Sendung entnommen wurle und frei ans dem Gesteine herauspräparirt werden lonnte. Dieselhen stammen zwar von derselhen Localität, gehören aber einem anderen, etwas grösseren Individunm an.

Das II aterial umfasst folgende Stücke:

1) Ob erschäd el (Taf. I $[X]$, Figs. 1 11. 2, Taf. Jl $[X I]$, Fig. 1) erhalten vom Condylus occipitalis his zum Vorderrande der Nasengrube, jedoch ohne Jochlogen uncl zum Thleil stark lädirt an der Crista und dem hinteren Fortsatz derselben. Ehenso sind Iurch Zerschlagen im Steinhrnch einige Zähne zerfallen nnıl verletzt. In Tebrigen aber ist der Erhaltungszustand von seltener Schönheit, und können auch die Knochennähte meist genan festgestellt werden. Der U'nterkiefer fehlt vollständig.

2) Wi rluel (Taf. II [XI], Fig. 2, Tal. IIl [XIJ], Fig. 1-9). Der Atlas fehlt, dagegen ist der Epistropheus der 3., 5. und 6. Halswirbel recht gut. die IIalswirbel 4 und 7 fragmentarisch erlalten. Aus der Rückenregion sind (f Wirbel ziemlich vollständig, währent die anderen theils nur in Bruchstücken vorliegen, theils ganz lehlen oder durch den zweiten Fund erginzt werden. Die Lendenwirbel liegen vollständig in mehr oder minder guten Stücken vor, ebenso ein Sacralwirbel. Leider fehlen the Schwanzwirbel vollständig, resp. konnten nicht aus dem Ciesteine heranspräparirt werien.

3) Riplen (Taf. HI [XII]. Fig. 10-12). In den Gesteinslï̈cken waren zway vielfach Rippenfragmente, vielleicht anch ganze Stücke erhalten, dieselben mussten aher bei dem Prïprarieren der wichtigeren Skeletstïcke geoflert werlen, so dass mur noch 6 proximale Endigumgen rorliegen.

Ueberllicken wir das ganze Material, so sehen wir, lass uns zwar sehr wichtige Skeletstücke, wie der Unterkieler, der Brust unl Beckengürtel mit len Extremitäten, die meisten Rippen und Schwanzwirhel ginzlich fehlen oder doch nur in schlechten Fragmenten vorliegen, alıer der erhaltene Rest ist immerhin so wichtig, zutlem da er mit des Ausnahme der 5 nachträglich gefundenen Wirbel von einem Individuun stammt, dass die Zusanmenstellnng desselben ein hesseres Bild des Thieres bietet, als wir es von den meisten frülıeren Zeuglodonten bekommen konnten.

\section{Dev Schäidel.}

Am Schädel fehlt, wie bereits erwähnt, die Schnanzenspitze von dem vorderen Endle der Nasengrube ab; sie hat die 3 Incisiven getragen und wurde in den Proportionen von Zmglodon Osiris auf der Abhildung ergänzt. Nach der ïbrigen Form und Gestaltung des Schädels sind diese Proportionen mit grosser Wahrscheinlichkeit anzunehmen, und die Ergänznng erschien mir nothwendig, mm das Gesammtbilı klar vor Augen führen zn kömnen. Bei den Mfassangaben sind diese Verhältnisse zu Grunde 
gelegt, jedoch mit einem ca. versehen, soweit keine Messung am Stücke selbst vorgenommen werden konnte. In der Ansführung der Tabelle schliesse ich mich möglichst an diejenige von Stromer (1. c. pag. 80) an und füge zur Erleichterung des Vergleiche mit Zeuglodon Osiris die dort angegebenen Maasse bei.

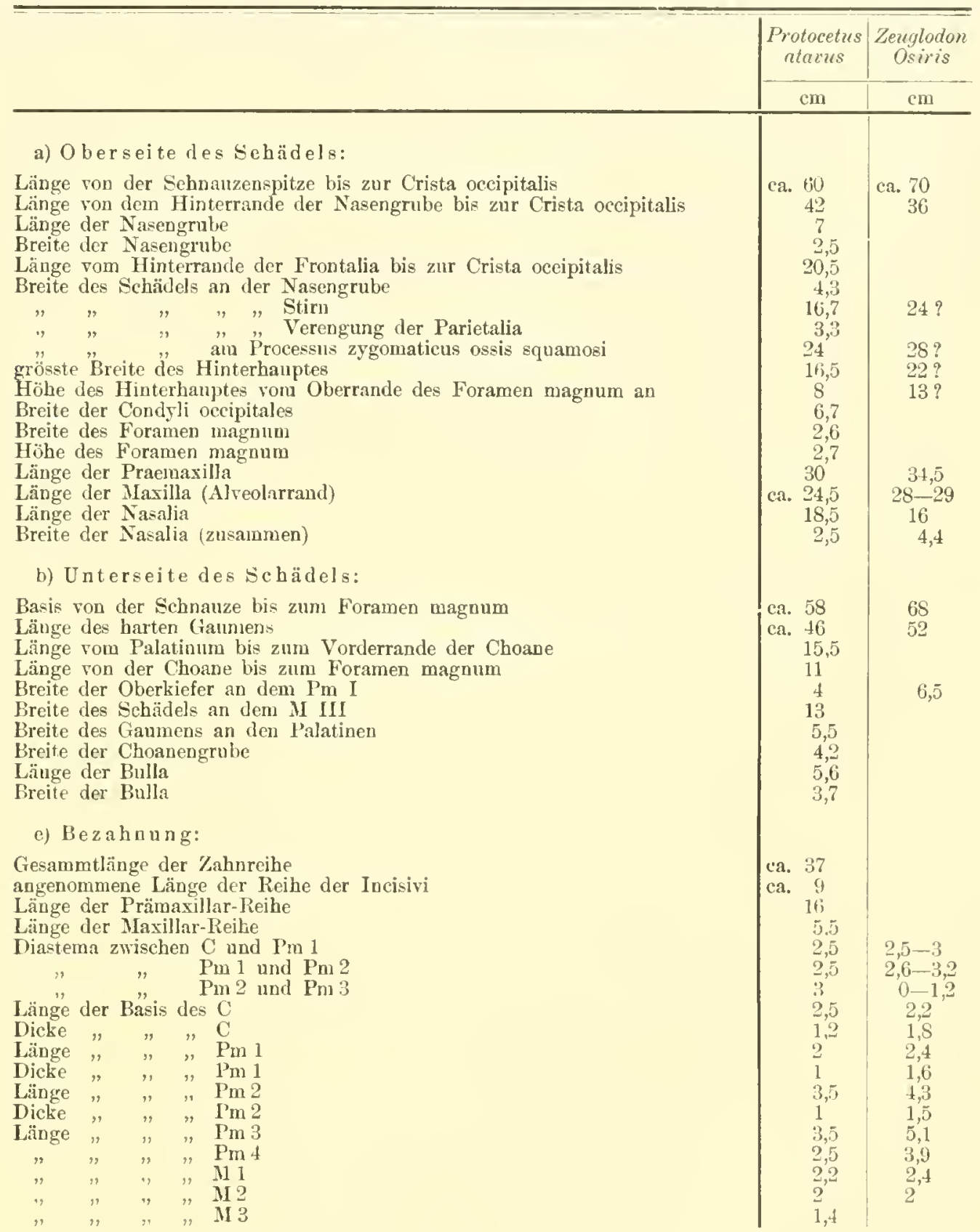

Die Tabelle sowohl wie ein Blick auf die Abbildung zeigt uns, dass wir einen relativ recht zierlichen Schädel eines Zeuglodonten vor uns haben, der in seiner Länge noch hinter dem STRomer'schen Exemplar um etwa 1/6 zurïcksteht, insbesondere aber bedeutend schlankere Gestalt anfweist. Es 
macht sich dies ganz besonder's an dem eigenthichen Schnanzentheile bemerkbar, der zwischen dem Pm ․․ und Pm 3 sich rasch verjüngt und dann als annähernd gleich schmales gestreckites Rostrum, soweit erhalten. nach vorue verläuft. Dementsprechend sind auch die daran theihnelmenden Skelettheile anssergewöhnlich schlank und gestreckt. Die Nasengrube. Welche bei anderen Zenglodonten nach vorn ohne eigentlichen Abschluss verlïuft, hildet hier eine wohlungrenzte Grube, deren Hinterrand rurch die Nasalia gebildet wird, während ter ührige Theil ron den Prïmaxillarien umschlossen wird. Eine mediane Scheidewand wurde nicht heohachtet. Yon besonderer Betlentung ist die Lage der Nasengrube, welche sich bedentend weiter vorne an der Schnauze befindet als bei allen anderen Zenglodonten. Wir kemnen zwar leider den Abstand ron der Schnauzenspitze nicht, sehen aber doch, dass der Hinterrand der Nasengrube mit dem Vorderrande des Pm 1 abschneidet, wälırend derselbe bei Zenglorlon Osiris erst am Vorderrande des Pm 2 und bei dem Schädel von Harle m (Z. cetoides Owen) noch mehr zurückliegt. Hiermit hängt anch rlie wesentlich grössere Länge der Nasalia zusammen.

In Folge der günsticen Erhaltung bekommen wir anch ein klares Bild ïber das Verlültuiss der Orbita zu dem grossen Schläfenansschnitte. Die eigentliche Orbitalgruhe ist auffallend klein, seitlich gelegen und wird gewissermaassen überdacht ron dem stark ausgebildeten Processus postorbitalis der Frontalia. Auf der Unterseite wird the Orbita umschlossen vom Jugale, ohne dass jedoch ein Boden fler Orbita gebildet wirl, ein Lacrymale kann ich nicht heobachten. Nach vorne endigt die Orbita ziemlich spitzwinklig, mach hinten ist sie offen; die Lünge rom Processus postorlhitalis zum Vorderrande beträgt $7 \mathrm{~cm}$, lie Höhe $11 u r 1,5 \mathrm{~cm}$.

Wie hei den ïbrigen Zenglodonten folgt hinter den breit anslegenden Flügeln der Stirnbeine eine ganz anffallende Verjüngung des Schädels in ter Parietahregion, die nu so stärker hervortritt, als zugleich die Oberkante in eine Crista ansgezogen ist, so dass der Querschnitt ein Dreieck bildet, dessen Seiten $9 \mathrm{~cm}$ bei einer Breite der Basis (Palatinum) von $n$ ur $5,5 \mathrm{~cm}$ messen. Die Seitenwände der ansserordentlich grossen Sehläfengrube sinł nur sehr dünn verknöchert, und zwar nehmen daran ein nach unten gerichteter linterer Fortsatz der Frontahia, the Parietalia und die offenbar sehr grossen Alisphenoide theil; zwischen diesen Knochenplatten und einem nach oben gerichteten Flïgel wes Pterygoides bleibt aber noch eine ziemlich grosse nuverknöcherte Stelle der Schädelwandnng. wie wir es auch bei vielen Pinnipertiern finden.

Stromer nimmt an, lass der Jochbogen bei den Zenglodonten nur durch einen diimen geraden Stab wie hei den Denticeten. gebihket werde. Ich kann mich dieser Ansicht nicht anschliessen, denn sowohl bei unserem Exemplare wie bei dem grossen Mesocetus Selnceinfurthi sind nicht unerhebliche Theile des Jugale erhalten, das sich als eine ziemlich hreite, abgetlachte, aber im Uebrigen normal ausgebildete Knochenspange darstellt, welche sich in keiner Weise mit dem diinnen gerarlen Knochenstab der Walthiere vergleichen lässt.

Gegeniiber der parietalen Verengung des Schälels ist die hintere Schä l elkapsel wieder etwas anfgetrieben und zeigt auch eine stärkere Verknöcherung, an welcher sich besonders ،lie Sıuamosa betheiligen. Die Crista ist weit nach hinten ansgezogen und bildet am Hinterrande ein stark rorspringendes, halbkreisförnnig gerundetes Dach. das uach hinten und oben gerichtet ist. An dieser Verlängerung der Crista scheint das Supraoccipitale nur wenig betheiligt zu sein, so dass sie im Wesentlichen dem Parietale orler vielleicht auch einem Interparietale zufällt.

Die Hinterhan uts e gion (Taf. II [XI]. Fig. 1) ist nahezu tadellos erhalten. Am auffallendsten ist die soeben erwïhnte Ueberdachung durch den hiuteren Fortsatz der Crista. Darunter liegt das fast 
senkrecht abfallende, dureh liräftige Muskelansätze ausgezeichnete Supraoceipitale mit den wohl ausgebildeten Condylen, welche das abgerundet-quadratische Foramen magnum umschliessen. Die seitlichen Flügel sind fest verwachsen mit dem grossen Perioticum, das sich an den mur wenig ansgebildeten Processus paroecipitalis anlegt. Die ganze Entwickelung des Hinterhauptes zengt ron einer überans kräftigen Nackenmusculatur, welche hier ansetzte.

Die Unterseite des Schüdels (Taf. I $[X]$, Fig. 2) giebt uns infolge günstiger Erhaltung Aufsehluss über manche bisher unbekannten Einzelnheiten. Der harte Gaumen ist uns zwar nicht bis zur Schnanzenspitze erhalten, aber doch bis zum Ansatz des Intermaxillare und lässt hier so wenig, wie bei dem Strouer'schen Exemplare, Durchbrïche erkennen, welche als vordere Gaumenlöcher zu deuten wären. Es ist wohl anzunehmen, dass der Gaumen vorn vollstänlig gesehlossen war, ebenso wie in dem ganzen durch die Maxillaria gebildeten Theile. In dem verlängerten Schnauzentheile ist der Gaumen median eingeseukt und bildet eine leichte Rinne, die sich nach hinten verflacht. In der Gegend des $\mathrm{Pm}_{4}$ beginnt das Palatinum, aber auffallenderweise bleibt nun der Gaumen nicht mehr gesehlossen, sondern die Palatina und der hintere Theil der Maxillaria sind getrennt dureh die Oefinung der Orbitalgrube. Hiedurch erscheinen die Maxillaria mit den 3 Molaren gleiehsam freistehend und gehen über in die sehmalen Kinochenspangen der Joehbogen. Die Palatina jedoch greifen noch weit zurüek und zeigen in ihrer hinteren Hälfte eine dureh eine scharfe Kante markirte Erhöhung, welehe in eine förnuliehe Crista übergeht. In diesem Theile nehmen auch noeh die Pterygoide an der Bildung des harten Ganmens theil, indem sie sich seitlich an die Palatina anlegen. Hiedurch wird die Choanengrube sehr weit nach hinten gedrängt, da der Gammen erst $11 \mathrm{~cm}$ vor dem Hinterrande des Schädels endigt. Die seitliche Umrandung der Choane wird dureh die hinteren Flügel der Pterygoide und vielleicht anch durch einen Theil der Alisphenoide gebildet, wïhrend an der eigentlichen Schädelbasis merlian das Basisphenoid und Basioceipitale sich betheiligen.

Seitlich an die Schädelbasis legt sich das grosse Squamosum mit der Articulation für den Unterkiefer an. Durch einen stark vorspringenden Processus postglenoidalis ist eine grosse, nach vorne geriehtete Gelenkgrube geschaflen, welehe aber doeh etwas flach und wenig ansgeprägt erscheint, wie wir es ja anch lianm anders bei der cetaceenartigen Gestalt des Unterkiefers und seiner hinteren Gelenkflïche erwarten können. Der Fortsatz gegen das Jngale seheint auf der rechten Seite vollstïndig erhalten zu sein und wäre demnach relativ kurz und gedrungen.

Besonders anffallend ist an der Unterseite des Schädels die wohlerhaltene Gehörregion, welche dureh eine ïberaus kräftige Auslildung des Tympanieums nnd Perioticums gekennzeichnet ist. Das Tympanienm bildet eine hoch aufgeblähte Bulla von äusserst harter, solider Verluöehernng und erinuert hierin, wie auch in der äusseren Form, am meisten an das hetreffende Organ der Bartenwale. Eine tiefe. noch mehr als bei Znuglodon Osiris ausgeprägte Furche verläuft von der Mitte des Hinterrandes quer naeh der Innenseite und zerlegt die Bulla in zwei ungleiche Theile, von welchen der kleinere in der hinteren Eeke auf der Innenseite als Wulst hervortritt. An der Vorderseite der Bulla liegen eine oder melrere Eintrittstellen ron Nerven, und dürfte wohl hierin das Foramen lacerum medium, vielleicht auch das Foramen ovale zu erblicken sein. Ebenso konnte der Meatus anditorius externus blossgelegt werden, der sich an Aussenrande der Bulla befindet und in die Rinne ansmündet. welche dureh den Processus postglenoidalis und das Periotieum gebildet ist. Hinter der Bulla tympani und fest mit dem Paroceipitalfortsatz verwachsen ist das Perioticum eingeschaltet. Dasselbe hat eine rauhe, complieirt gestaltete Oberflïche mit mehreren Buckeln und Höekern und ist am besten aus ler $A b-$ Geolog. u. Palaiont. Abh., Y. F. VI. (der ganzen Reihe X.) Bul.. Ufeft 3. 
bildung (Taf. I $[X]$, Fïg. 2) ersichtlich. Zwischen dem Perioticum und der Bulla ist das Foramen lacerum posterins wohl ausgelüigt. Ebenso' ist zwischen dem Perioticum und Basisnccijitale ein tiefer ovaler Schlitz offen, den auch schon J. MüLLER und Stroxer beobachten komuten und der wohl als Foramen condyloidem angesprochen werden dart.

Die Bezahnnng ist bei unserer Art so verschiedenartig ron allen bis jetzt bekannten Zenglodonten ansgebildet, dass sie das Hauptcharacteristicum billet. Sie ist $\mathrm{nm}$ so interressanter, als sie gegenüber den anleren Zenglodonten einen ausgesprochen primitiven Charakter trägt und uns dadureh einen Hinweis anf die Stammesgeschichte der Zenglodonten bietet. Die Zahuformel $\frac{3.1 .4 .2}{3.1 .4 .3}$, welche Stroner von Zeuglodon Osiris giebt, halte ich für richtig, obgleich anch an meinem Exemplar kein Zahnwechsel $\mathrm{zh}$ beohachten ist und so die Frage über das Verhältniss der Molaren und Prämolaren nielt absolnt sicher zu entscheiden ist. leh zweifle nicht, dass anch unser Typus 3 Incisiren besessen hat und dass der vorderste erlaltene Zahn den Canin darstellt, wie ja anch ans der Naht zwischen Maxillare und Praemaxillare herrorgeht. Darans ergieht siclı für Protocetus die Zahnformel $\frac{3.1 .4 .3}{\text { ?(3. 1. 4. 3) }}$ Ganz entsprechend dem Schälelban weist die Stellung der Zähne im vorderen Schmanzentheile eine ausgiebige Streckung anf, gegenïber der gedrungenen und engen Zahnstellung in hinteren Tlieile des Gebisses.

Die : Incisiven werden wohl, wie bei Zenglodon Osiris, einfache nnd einwurzelige léegelzähne gebildet laben. Der Canin ist leider nur auf der rechten Seite erbalten und anch da an der Zahnkrone lälirt. Ls lässt sich aber erkennen, dass der Zahn sehr kräftig gehant war und abweichend ron Z. Osiris eine langgestreckte Zahnhasis aufweist, welche in der Nitte etwas eingezogen ist, ohne dass es jedoch zur Ansbildung von 2 Wurzeln gekommen wäre. Der Sehmelz ist auf der Aussen- mo Innenseite stark runzelig, an der Vorderseite ist eine scharfe liante, an der Basis auf der Anssenseite ein kräftiger Wulst entwickelt.

$P m_{1}$ ist anffaltend klein nnd schwach gebaut, gleichfalls mit einfacher Zalnkirone, die jedoch nach hinten etwas:ausgezogen ist. Auch dieser Zahn ist im Terhältniss zur Breite sehr lang (2:1), und die Zahnwurzel ist wie hei $Z$. Zitteli und dem Harlemer Schädel zweiwurzelig. Der Zahmschmelz ist glatt und mit einer leichten medianen Kante versehen.

Wihrend nun hei den ibrigen Zeuglodonten die charakteristischen Zackenzälne beginnen. ist der Pm., ebenso wie die folgenden, vou durchans verschiedenem Typus. Er ist sehr kräftig gebaut, mit langgestreckter, zweiwureliger Basis, einer grossen vorleren Zahnkrone und einem kleinen hinteren Höcker. Der Schmelz ist wie am Canin kräftig gefaltet und sowolnl an der vorderen Spitze wie an dem hinteren IIücker angekaut. Eine mediane liante länft über die Vordel- und Hinterseite der IIanptspitze.

Während his zmm $\mathrm{Pm}_{3}$ zwischen den Zälunen weite Lücken frei bleiben, beginnt mit diesem die Serie der fest geschlossenen Zahnreihe. $\mathrm{Pm}_{3}$ ist der kräftigste Zahn des Gehisses; die Zahmbasis ist nicht einfach langrestreckt wie bei den vorderen Zähnen, sondern anf der Innenseite in der hinteren Hälfte rerdickt, indem die hintere Wurzel viel dicker entwickelt ist als die rordere; vielleicht war dieselhe sogar gegabelt, doch ist dies nicht sicher zu entscheiden. Die Zalnkrone spitzt sich in einem grossen, in der Mlitte gelegenen Zahnkegel zn, der sowohl auf der Spitze wie an Vorderrande stark ahgekant ist. Ansserlem ist hinten noch ein kräftiger, gleichfalls angekauter Höcker ansgebildet. Der Sclmelz ist glatter als an dem Pma und zieht sich etras an der Innenseite an der nach innen gestellten Zahnwurzel herunter. 
$\mathrm{Pm}_{4}$ ist etwas kleiner als $\mathrm{Pm}_{3}$, aber von demselben Ban, nur ist die Hanptspitze mehr naeh rüekwärts und innen gebogen, und der Sehmelz reicht viel tiefer an der dritten inneren Zahnwurzel herunter. Die Spitze und Vorderkante ist stark angekant, der hintere Höcker jedoch nicht.

Die folgenden $\ddot{\partial}$ Molaren sind auffallend klein und liegen dieht gedrängt anf der Innenseite des hinteren freien Kieferfortsatzes, denn der knöeherne Theil des Gaumens schliesst nur bis hinter den $\mathrm{Pm}_{4}$ an das Maxillare an. Diese Zähne standen oftenbar nur in losem Verbande mit dem líeferknochen und wurden melı durch lräftige Ligamente als dureh tiel eingesenkte Alveolen festgehalten. Es ist kein Zweifel, dass sieh hier bereits eine Rüelibildung anbahnt, wie sie auch ron STroner ganz riehtig angenommen wurde. $\mathrm{MI}_{1}$ ist nur auf der linken Seite erhalten und anch hier ansserordentlich stark abgekaut. Der Zahn war offenbar zweihöekerig, und zwar derart, dass die vordere Zahnspitze etwas kräftiger als die hintere war. Auf der Innenseite zieht sich der Sehmelz weit herunter, ohne dass jedoch noch ein Absatz oder Innenhöcker zu beobachten wäre. $\mathrm{I}_{2}$ ist weniger angehaut und zeigt ausser der mittleren Hauptspitze und dem hinteren Höker noch einen schwachen vorderen Höcker; auch hier ist ter Schmelz auf der Innenseite ïber die dritte nach innen gestelte Zahnwurzel tief herabgesenkt. $\mathrm{M}_{3}$ ist klein. ansgesprochen dreiwurzelig, mit einer Hauptspitze und einem kinen vorderen Höcker, der dadureh entstanden ist. dass der untere Wulst an der Aussenseite der Selimelzkappe am Vorderrande nach oben bis in die Nähe der Spitze gezogen ist. Auf der Innenseite greift der Schmelz wiederum tief an der inneren Iritten Zahnwurzel hinnnter. Der letzte Molar seheint fast frei in der Luft zu stehen und hat nu ganz wenig Verband mit dem an dieser Stelle bereits als sehmale Leiste ansgebildeten Kíefer. Die Zahnreihe endigt dicht vor der Naht zwischen Maxillare und Jngale.

Gestützt anf diesen mehr beschreibenden Theil des Schädels gehen wir über zu der V'ergleichng unseres Typus mit anderen und zu den allgemeinen Schlïssen. welche wir ans dem Schäılelbau mores Exemplares gewinnen kömnen.

Die klare Darstellung von Stromer ïber den Aufban des Zeuglodontenschädels entlebt mich einer ausführlichen Zusammenstellung, indem sich auch unser Typus in seinem allgemeinen Anfhau rollstïndig an den der ïbrigen Zenglodonten angliedert. Eine Frweiterung unserer lienntnisse lahen wir jedoch in mancher Hinsicht gewomen. Ahgesehen von den Besonderheiten unseres Typus werden wir in Folge günstiger Erhaltung über the Ansbildung und Betheiligung der einzelnen Skeletelemente vielfach aufgeklärt. Der Aufl,au der Schnauze war schon früher bekannt, ebenso die eigenartige Gestalt der Frontalia mit dem weit vorspringenden Processus postorbitalis; neu ist dagegen der Bau des hinteren Sclädeldaches, an welchem las Parietale einen verhältnissmässig kleinen Antheil nimmt, aber durch die Austildung der Crista besonders hemerkenswerth ist. Ganz auffallend ist dahei das grosse Squanosum, das gänzlich von dem der Denticeten abweieht und viel eher mit dem der Pinnipedier, noch mehr aber mit dem iler Carnivoren verglichen werden kann. Dasselhe gilt anel von der Lage und Ausbihung des Alisphenoides und dem nach oben greifenden Flügel des Pterygoides. Betrachten wir den Ban der Schädelkapsel im Cranzen, so miissen wir zugestehen, dass derselbe in allen ron den der Denticeten versehieden ist und sich anch nicht mit dem der Mysticeten in Einklang bringen lässt. Bei den uns bekannten Arten der Pinnipedier ist das Gehirn stets anffallend gross und dementsprechend die Auftreibung der Gehirnkapsel eine so ausgesprochene uni vollkommen ausgeliddete, dass uns anch hier eine Angliederung schwer fällt. Suchen wir naeh einem Vergleich, so werten wir unbedingt an die Carnivoren nud speeiell an die Caniden oder richtiger an deren alten Vertreter unter den Creodontiern anknüpfen müssen, dem hei diesen finden wir in auffallender Weise alle die Verhältnisse 
in der Gestalt und dem Anfbau der Schädelkapsel wieder, ja selhst die charakteristische Ausbildung der Frontalia ebenso wie die scharfe Crista ist bei diesen in analoger Weise angelegt.

Die Unterseite des Schälels ist gleichfalls noch nie so gut hlossgelegt worden wie bei unserem Exemplar uml sie beweist uns, dass der harte Gaumen sehr weit nach rïckwärts greift olne vordere Gammendurchbrüche. An der Gaumenbildung betheiligen sich nur in vorderen Schnauzentheile die Maxillaria und Praemaxillaria, während der ganze hintere Theil dureh die Palatina und die nach innen gekehrten Flügel der Pterygoidea gebildet wird. Die Choanengrube ist dadurch sehr weit nach hinten bis an das Basisphenoid gerïckt. Die grossen, etwas ausgeflachten Articulationsflächen für den Unterkiefer stehen rechtwinklig von der Medianlinie ab und werden durch einen kräftigen Processus postglenoidalis gebildet. Die Gehörregion ist sehr kräftig entwickelt mul yor allem durch das anfgeblähte Tympanicum ansgezeichnet, an welches sich jedoch noch ein gleichfalls grosses Petrosum angliedert, das sich mit dem Occipitalfortsatz zı einem kräftigen Processus mastoidens vereinigt. Der Meatus auditorius mündet seit wärts nach anssen. Das Hinterhaupt ist anffallend lküftig und durch Ansatzstellen starker Musculatur ausgezeichnet.

Es ist nun nubedingt zuzugeben, dass die langgestreckte Schnanze und der weit nach hinten reichende harte Gaumen in seiner äusseren Form eine Analogie bei den Denticeten hat, die sieh gleichfalls dnrelı eine Streckung der Schnauze auszeichnen. Fassen wir aber den Aufbau des Gaumens näher in das Auge, so fallen uns sofort auch die Unterschiede anf. Bei den Delphinen wird der Gaumen fast ausschliesslich Inrch die Kieferknochen gebildet, an welehe sich im hinteren Theile ein kurzes Palatinum und die Flügel ler Pterygoile anschliessen; bei den Zenglodonten dagegen ist las Palatinum gross und gewissermaassen normal wie hei den Carnivoren gebildet und hat in Lage und Form mit lem der Denticeten nichts gemein. Dass die Choanenüffnung sehr weit zurïckliegt, wird allgemein als eine Einrichtung angesehen, welche allen wasserbewohneuden Thieren zukommt und die sich ans dem Bedürfniss erklärt, die Luftwege möghichst abzuschliessen, um ungehimlert die Nahrung im Wasser aufnehmen zu können. Wir hahen es also dahei nur mit einer Convergenzerscheimmg zu thun, die aus natülichen Beduirfnissen hervorging. Hieraus erk]ärt sich auch der Mangel anderweitiger Oeffnungen im Gaumen zur Genüge. Würden die Denticeten von den Zeuglodonten ahzuleiten sein, so mïssten wir, eine naturgemässe Eutwickelung voransgesetzt. eher eine Vergrösserung als eine Verkïrzung der Palatina finden, was aber, wie erwähnt, nicht der Fall ist. Eine Analogie zeigen nus die Pterygoide, welche hier wie dort breite Flügel entwickelt liahen, die median znsammentreten, un so zur Verlängerung des geschlossenen Gaumens beizutragen. Anch hierin sehe ich aber nur eine Convergenzerscheinung, hervorgegangen aus dem Bedürfniss, die Choanenöffnnmg möglichst abzuschliessen. Sehr charaliteristisch ist die eigenartige Crista am Ende der Palatina, welche darauf hinweist, dass ursprünglich der Caumen lier endigte, und zwar mit einer ähnliclıen Verdickung, wie wir sie hei den Carnivoren, speciell den Creodontiern zuweilen beobachten, und dass die Aushildung der Pterygoide erst eine secundäre Erscheinung ist.

Das hoch anfgetriebene Tympanicum lat allerdings am meisten Aehnlichkeit mit der Bulla der Walthiere, speciell der Mysticeten, nicht der Denticeten, aber wir dürfen nicht ausser Acht lassen, dass eine analoge Bildung anch bei den Landthieren luäufig ist und dass die kriftige Ausbildung des Perioticums leineswegs mit dem der Mysticeten, noch weniger mit dem der Denticeten sich in Einklang hringen lässt, sondern eine Analogie bei den Carnivoren finlet. Leider sinıl uns diese Schỉdelparticen vou den in Frage kommenten Creobontiern nur wenig bekannt, und ich kann mich unr auf die mïnlliche Mittheilung von Prof. W. B. Scott ans Princeton N.J. herufen, der hierin eine volle Analogie mit den ilm bekannten Verhälnissen bei Inyunodon erkennen wollte. Jedenfalls finden wir bei den 
lebenden Caniten und bei deren fossilen Vertretern Galecyms und Temmocyon nach den Angabeu von Cope eine vollkommene Analogie in der Ausbildung der Gehörregion. Ganz verschieden und nicht in Einklang zu bringen ist die Ausbildung des Ohres bei den Pinnipediern, bei welchen Tympanicum und Perioticum gleichsam zu einer grossen gemeinsamen Bulla verschmilzt, welche in der Mitte stark eingezogen ist. Ein Verschmelzen mit dem Processus paroccipitalis und mastoideus wie bei Zeuglodon findet weder bei den Walthieren noch bei den Pinnipediern, wohl aber bei den Carnivoren statt. Eigenartig scheint nur die ausserordentliche Festigkeit und Dicke des Knochens der Bulla zu sein, und dies mag wohl wiederum als eine charaliteristische Eigenschaft der Wasserbewohner zu betrachten sein, bei welchen natïrlich in Folge des läutfig und stark schwankenden Druckes die zarten und empfindlicheu Gehörorgane einen ganz anderen und besseren Schutz verlangen als bei den unter gleichmässigem Atmosphärendruck lebenden Landthieren.

Das Hinterhaupt mit seiner markanten, scharf abgesetzten Gestalt und den kräftigen, weit vorspringenden Condylen weist auf den Ansatz sehr kräftiger Nackenmuskeln und grosse Beweglichkeit des Schädels hin und steht dachurch in scharfem Contrast zu den weichen, gerundeten Formen bei den Walthieren, bei welchen die grossen flachen Condylen kaum ans dem Schädel heraustreten. Es lässt sich viel eher mit dem der Pinnipedier vergleichen, bei welchen ja anch der liopf sehr frei in der Bewegung und der Hals wenig relucirt ist. Die Grösse der Schädelkapsel verlangte bei dieser Gruppe jedoch eine Unformung des Hinterhauptes, das sich demgemäss bedeutend höher und gerundeter darstellt als bei den Zeuglodonten. Auch hier finden wir bei den Carnivoreu mit ihrem dreieckigen abgeflachten Hinterhaupt und den kräftigen, hochgewölbten Condylen die schönste Analogie.

Das grösste Interesse beansprucht mit Recht die Bezahnung. Bei unserem Typus weicht dieselbe allerdings stark ab von derjenigen der ïbrigen Zeuglodonten, aber wir diurfen die Besonderheiten derselben mit Sicherheit als e in atavistisches Stalium des echten Zenglodontengebisses bezeichnen. Wir sind deshalb wohl berechtigt, gerade unseren Typus zum Ausgangspunkt der vergleichenden Untersuchungen zu machen und daran erst die spätere Differenzirung anzuschliessen.

Die Zahnformel $\frac{3 .}{3 .} \frac{1}{\text { (?) } 3.1 .4 .3}$ weist anf die Abstammung von Thieren mit vollständig entwickeltem Gebisse hin. Die 3 Incisiven nehme ich als einfache Kegelzähne, wie bei den ïbrigen Zenglodonten, an. Ob dleselben unter sich und gegenüber dem Canin unterschiedlen waren, lässt sich nicht entscheiden. Jedenfalls unterscheidet sich aber der Canin gegenüber den nachfolgenden Pm, durch Grösse und die einfache Wurzel. Die Prämolaren sind unter einander verschieden, indem der Pm, klein, zweiwurzelig, mit einem Höcker, der $\mathrm{Pm}_{2}$ gross, zweiwurzelig, mit 2 Aussenhöckern, Pmo sehr gross, zweiwurzelig, mit verdickter hinterer Wurzel, 2 Aussenhöckern, $\mathrm{Pm}{ }_{4}$ gross, dreiwurzelig, mit 2 Aussenhöckern und tief nach innen verlängertem Schmelz ausgebildet ist. Alle Molaren und Prämolaren sind ansgesprochen secontont. Es ist wohl gerechtfertigt, anzunehmen, dass die eigeuartige Verlängerung des Schmelzes nach der inneren Zahnwurel nur als eine Rïckbildung aus cinem früheren Innenlı̈oker anzuselien ist, der bei $\mathrm{Pm}_{3}$ nur leicht angedentet, bei $\mathrm{Pm}_{4}$ dagegen wohtentwickelt war. $\mathrm{Pn}_{4}$ ist kleiner als $\mathrm{Pm}_{3}$ und in keiner Weise als Reisszahn ausgebildet und den anderen gegenüber differenzirt. Die vorderen 2 Molaren schliessen sich in ihrer Ausbildung ganz an $\mathrm{Pm}_{4}$ an und sind wie dieser aus tritubercularen Zähnen hervorgegangen. $M_{3}$ ist klein, mit einem kräftigen Aussenhöcker und imnerer Schmelzlage, die auf einen gleichfalls kräftigen Inneulı̈cker zurïckzuführen ist. Alle Nolaren sind dreiwurzelig. Vergegenwärtigen wir uns diese Zähne in ihrer ursprünglichen Gestalt, 
indem wir an Stelle der Schmelzlappen uns Innenhöcker vorstellen, so haben wir ein typisches Raubthiergebiss ror Angen, dessen Anschluss an bekannte Arten nicht allzuschwer fällt. Der Mlangel eines Reisszahnes schliesst die echten Carniroren oder Fissipedier aus und rerweist uns an die Gruppe der Creodontier, an welche wir ja anch aus geologisehen Gründen zu denken haben.

Es wäre wohl zu weit gegangen, wollten wir eine bestimmte Art der Creodontier als Ausgangspunkt für die Zenglodonten bezeichnen, denn einerseits ist denn doch unsere Kenntniss dieser alten Raubthiere eine reclit dürftige und deshalb auch die Systematik derselben nur als eine prorisorische anzusehen, anderseits sind die Merkmale der ursprüngliehen Form auch bei Protocetus schon so sehr verwischt. dass wir nicht mehr die feinen Interschiede feststellen können, welche als Unterscheidungsmerkmale der einzelnen Creodontierarten erforderlich wären. Wir mïssen uns damit begnïgen, den allgemeinen Typus nachzuweisen, und maassgebend hierfür erscheint mir erstens der ausgesprochen secodonte Charakter des Gebisses, zweitens die lückenlose Vollständigkeit 3. 1. 4. 3, die wir als primitiven Typus bezeichnen kömnen, drittens die gleichmässig trituberenlare Ausbildung der Molaren und des $\mathrm{Pm}_{4}$, sowie die Andentung eines kleinen Innenhöckers bei Pm „. Dem secodonten Charakter würden am meisten die Hyänodontiden gerecht, aber bei diesen fehlt bereits $\mathrm{I}_{3}$, anch lïsst sich der Ban ron $\mathrm{Pm}_{3}, \mathrm{Pm}_{4}$ und den Molaren nicht gut in Einklang bringen; dasselbe gilt von den Paläonictiden, wogegen wir bei den Proviveriden (Schlosser) Eigenschaften im Zahnban wie in der Zahuformel finden, auf welche sich unser Protoctus: ohne grossen Zwang zurückführen liesse. Ich möchte dahei ganz speciell auf die als Simopr Leidy (Stypolophns Cope) hesehriebenen Arten hinweisen. welehe fast in jerler Hinsicht unseren Anforderungen an eine Lrform für die Stammreihe der Zenglodonten gereeht werden. Freilich liegt mir ferne, gerade diese Art als Ahnen zu bezeichnen, sondern es soll damit nur der Typus angerleutet sein, an welchen wir als Stammform zu denken haben. Vielleicht ist es nur zufällig, aber jedenfalls nicht uninteressant, dass der von Cope. (The Vertebrata of the tertiary Formations of the West, Rep. U. S. Creol. Survey of the Territories. 189t. Vol. 3) beschriebene und t. 2.5 of f. 1 abgebildete Schälel von Stypolophns whitiaf anch in seiner Stirnlinie eine auffallende Abflachung mit weit ausgezogenem Hinterhaupte mul kräftiger Crista zeigt. Wir sehen, dass wir aus dem Gebisse von Protortus Schlüsse auf die Stammformen zu ziehen gezwungen siml, die rollständig mit den übrigen Beobachtungen an Schädel übereinstimmen und in Einklang zu bringen sintl.

Die weitere Umwandlung les Gehisses ron Protoctus zu dem der cehten Zenglodonten ging offenbar rasch vor sich und folgte einem Gesetze, das wir anch bei anderen Seesängern finden. Die Zähne werden indifferent, indem sowohl der sectoriale Charakter, als auch die bestimunte Anordnung der Höcker verloren geht. Entspreehend der Lebensweise verlieren die Zähne die Function des Zerbeissens und Zerreissens der zälıen Fleischnahrung, sondern die Hantfunction wird anf das Ergreifen und Festhalten der Bente gerichtet, da für diese Arbeit die Extremitäten in Wegfall gekommen sind. Das Gehiss wird in einen Rechen ungewandelt, und die Zähne bilden sich theils in einfache Hakenzähne oder in Zackenzähne um. Wir kömen diese Erscheinung bei den Pinnipediern sehr hübsch verfolgen und finden sie ebenso bei den Denticeten, bei welehen allerlings ausser Squalorlon der einfachste Typus des Rechens mit homöodonter Zahnreihe vorwiegt. Die Unwandlung bei Zeuglonlon Osiris ist hereits in der Art rorgeschritten, dass Pm, zum echten Hakenzahn, die übrigen zu Zackenzähnen geworden sind, und zwar erscheinen die Prämolaren 2-4 mit hräftiger mittlerer Spitze, wälrend bei den Molaren die Hauptspitze mehr nach rome gerückt ist. $\mathrm{M}_{3}$ ist hereits rolständig reducirt und in 
Wegfall gekommen. Wie sich die Verhältnisse hej den grossen amerikanischen Arten gestaltet haben, ist nicht zu entscheiden, da das beschriebene Material nicht ausreicht.

Jedenfalls dürfte unser Protocetus im Vergleich zu Zeuglodon Osiris erwiesen haben, dass das Zenglodontengebiss genetisch in keine Beziehung zu dem der Pinnepedier und auch nicht dem der Squalodonten gebracht werden darf, sondern dass hier eine Convergenzerscheinung vorliegt. die sich aus der Lehensweise der Seesäuger ergiebt.

\section{B. Die Wirhel und Rippen.}

Der Atlas ist leider nicht erhalten.

Der Elistropheus (Taf. II [XI], Fig. 2-5) ist sehr bemerkenswerth, da er sich in recht gutem Erhaltungszustande befinclet. Die Maasse sind:

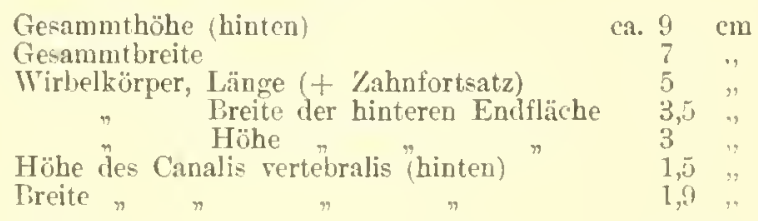

Die Abbildungen entheben mich einer eingehenden morphologischen Beschreibung und lassen die Eigenarten dieses Wirbels dentlich erkennen. Vor allem fällt der kiäftige, zapfenförmige Zahnfortsatz (Pr. odontoidens) auf, der auf seiner Oberseite nicht mu keine Rinne. sondern im Gegentheil eine wulstige Kante bildet und ron zwei flach gewölbten, etwas nach rückwärts gencigten Gelenlfacetten Hankirt wird. Es kann demnach ron einem Anklang an den Epistrophens der Wiederkïuer, wie dies Stroner (l. c. pag. 94) anführt, nicht die Rede sein, sondern wir liaben den Typus der Raubthiere vor nus. Entsprechend dem kurzen mud gedrungenen Bau des Halses ist auch der Epistropheus kurz, und den gedrungenen Ban entspricht auch die kräftige, aber verkürzte Diapophyse, deren obere und untere Wurzeln einen grossen Arterienlianal umschliessen. Eigenartig ist der hochgestellte Processus spinosus, (len auch LuCAS ${ }^{1}$ ) an einem amerikauischen Zeuglodonten beobachtet hat. Er unterscheidet sich von dem langgestrecliten Dornfortsatz der meisten Landraubthiere wohl, findet aber ein gutes Analogon bei den wasserlebenden Formen, wie Enhydra. Lutru. Castor filher u. a. ${ }^{2}$ ), in gewisser Hinsicht anch bei den Robben, bei welchen im Uebrigen der Ban des Epistrophens durch die geringe Aushildung der Diapoplysen und die weit rïckgreifenden Zygapophysen ein recht verschiedenartiges Gepräge hat, das sich noch mehr bei den Delphinen und Walen mit ihrer starken Reduction der oberen Bögen und Seitenanhänge kundgiebt.

Die übrigen Halswirbel (Tafel II [XI], Fig. 6-9) zeichnen siclı ebenso wie der Epistropleus durch kurzen mol gedrungenen Ban aus, was auf einen zwar sehr kräftigen, aber kurzen Hals hinweist. Bei allen sind ebenso wie an Epistropheus und, wie ich voransschicke, auch an den übrigen uns erhaltenen Wirbeln die Gelenlifacetten der Wirbelkörper vorn und linten fest verwachsen, ein nicht unwesentlicher Unterschied gegenïber den Pinnipediern und Walthieren. Die vorleren und linteren Zygapophysen sind stets deutlich ausgeprägt, die Arterienkanäle bis zum 6. Halswirbel wohłumschlossen als Oeffinungen zwischen den Wurzeln der Diapophysen.

1) American Naturalist. 1895. pag. 740 .

2) Vergl. E. STromer. Die Wirbel der Landraubthiere, ihre Morphologie und systematische Bedeutung. Zoologica. Heft 36. stuttgart 1902 . 
Die Maasse ergeben Folgendes:

\begin{tabular}{l|l|l|l|l|l}
\hline Vertebra cervicalis & 3. & 4. & 5. & 6. & \%. \\
\hline
\end{tabular}

Die wohlerhaltene Vert. cerv, 3 ('Taf. II [XI], Fig. 6 und 7) zeiclmet sich dureh den Mangel eines Dornfortsatzes und durch die Länge der seitlichen Fortsätze aus, inden sich die Diapophysen und Parapophysen zu einer Lamina perpendicularis verbinden, die schief zur Axe des Wirbels gestellt ist nud als sichelförmiger Fortsatz nach hinten und aussen ragt. Der heim Epistrophens noch scharf ansgehildete Grat auf der Unterseite des Wirhelkörpers verflacht sich bei diesem und dem folgenden Wirbel und ist bei Vert. cerv. 5 kaum mels angedentet. Vert. cerv, 4 ist mur als Fragment erhalten, gleicht aber in der Ansbildung der Seitenfortsätze ganz der Taf. II [XI]. Fig. 8 abgehildeten Vert. cerv. 5. Bei letzterer stellt sich bereits eine schwache Spina dorsalis auf dem zierlich gebauten oberen Bogen ein. Vert. cerv. 15 (Taf. II [XI], Fig. 9) ist durch die kräftige Entwickelung einer Lamina perpenlieularis ausgezeichnet. welche mur wenig nach hinten und aussen, in der IIauptsache nach unten gericlitet ist. Das Fragment der Vert. cervic. 7 lässt nur erkennen, dass der Wirbelkörper noch sehr zierlich ist und dass keine Parapophyse, sondern nur noch eine Diapophyse entwickelt ist, wie es anch sonst diesem Wirbel zukommt.

Die Rui ckenwirbel (Tal. III [XII], Fig, 1-4) sind leider vicht vollzählig erhalten, denn es liegen von unserem Exemplar nur \& Stïcke vor, doch bieten theilweise die später gefundenen Wirbel eine Ergänzung, da sie vielfach die Lücke ansfüllen. In der Tabelle wurden dieselben in Klammern gesetzt; sie rühren von einem etwas stärkeren Thiere her, doch glaube ich nicht, dass eine andere Species vorliegt. Vor allem fallen die Rückenwirbel durch ihre verhältnissmässig geringe Grösse und ihre wohlentwickelten oberen Bögen mit lohen Dornfortsätzen auf. Die Wirhelkörper sind solid verknöchert und zeigen keine Epiphysen an den flach amphicölen Gelenkflächen. Die Länge der Wirbelhörper nimmt stetig von rorne nach hinten zu, ebenso wie rie Breite bis zum 7. oder 8. Brnstwirbel. (Die $Y$, th. 7 ist seitlich rerdrürkt.) Die Breitenzunahme erstreckt sich jedoch nur auf den Oberrand

\begin{tabular}{|c|c|c|c|c|c|c|c|c|c|c|c|c|}
\hline Vertebrae thoracales & 1. & 2. & 3. & 4. & $\overline{5}$ & 6. & 7. & 8. & 9. & 10. & 11. & 12. \\
\hline Höhe von der Basis bis zur Spitze des & en & (n) & $\mathrm{cm}$ & cm & $\mathrm{cm}$ & $\mathrm{cm}$ & $\mathrm{cm}$ & & & $\mathrm{cm}$ & $\mathrm{cm}$ & $\mathrm{em}$ \\
\hline Dornfortsatzes & $-\ldots$ & ca. 12 & - & ca. 15 & - & - & 10,3 & & & (ca. 12) & $(11,5)$ & - \\
\hline Grösste Breite an den Diapophysen & 8 & 8,5 & 8 & 7,4 & ca. 7,5 & 7,5 & ca, 6,5 & & & (8) & $(7,5)$ & 5,2 \\
\hline Länge des Wirbelkörpers (basal) & 2,5 & 2,7 & 2,8 & - & - & ca. 2,8 & 3 & & & $(3,5)$ & $(3,5)$ & 3,5 \\
\hline Breite " , $\quad$ (hinten) & 4,2 & 4,7 & 4,8 & -- & 一 & 4,8 & 4,3 & & & $(5,5)$ & $(5)$ & 4,3 \\
\hline Höhe " , " , , & 2,7 & 2,5 & 2,7 & - & ca. 3 & 3 & 3,2 & & & $(3,7)$ & $(3,5)$ & 3,2 \\
\hline Höhe des Canalis vertebralis hinten & 2,2 & 1,8 & 1,6 & 1,5 & ca. 2 & 1,9 & 2 & & & $(2,3)$ & $(2,5)$ & 2,2 \\
\hline Breite , , " " " " & 2,3 & 2,5 & 2,7 & 2,8 & เล. 3 & 2,8 & 2 & & & $(2,7)$ & $(2,7)$ & 2,4 \\
\hline Höhe des oberen Bogens ", & - & 9,5 & $=$ & 11 & - & - & 7 & & & (ca. 8,5$)$ & $(7,5)$ & $\overline{5}$ \\
\hline Breite an den Präzygapophysen & 6 & 5,6 & 5 & 4,2 & - & & 3 & & & $(6)$ & (7) & 5,7 \\
\hline Breite an den Postzygapophysen & 5 & 5 & 4 & ca. 4 & - & 3,5 & 3,2 & & & $(5)$ & $(4,5)$ & 4,4 \\
\hline Länge des Dornfortsatzes (vorn) & - & ca. 8,5 & - & 10,8 & - & - & 7 & & & (ca. 7,5) & $(7,5)$ & - \\
\hline Mittlere Breite des Dornfortsatzes & 1,7 & 1,6 & 1,6 & 1,8 & - & - & 2,3 & & & $(2,5)$ & $(3)$ & ca. 3 \\
\hline
\end{tabular}


des Wirhelkürpers, wodurch die Gelenkitläche eine breite herzförmige Gestult hekommt. Auf der. Tnterseite sind die Wirhelkärper gerunlet, erst an der V. th. 11 und 122 ist eine leichte, nach hinten divergirende Doppelleiste entwickelt.

Die Diapophysen sind ansserordentlich kräftig an den vorderen 10 Brustwirbeln und tragen dementsprechend gegabelte zweiköpfige liplen. Erst am 11. Brnstwirhel verschwinlet die Diapophyse als seitlicher Fortsatz und geht in eine nach dem Wirbelkörper gestellte Querleiste über, währemd an dem darauf folgenden Wirbel nur noch eine oben und unten von einem Wulste hegrenzte raulıe Grube zur Aufnahme der offenbar einküpfigen Rippe ibrig bleibt. Ton besonderem Interesse ist die Entwickelung der Dornfortsätze, welche in keiner Weise an einen Meeresbewohner, sondern an ein echtes Landraubthier erinnern. Soweit erlalten, sind hieselben an den vorderen Rückenwirbeh sehr lang und schlank gebant; bei den ersten Wirbeln noch ziemlich steil gestellt. nelnmen sie linten eine immer stärkere Neigung nach rüickwärts an, die etwa an der V. th. 6 u. 7 melır als 45 Grad zur Verticalaxe des Wirbels beträgt. Bei den letzten 4 Rückenwirbeln stehen die Dornfortsätze, die rasch an Breite zu- und an Länge abnehmen, senkrecht zur Längsaxe, und es entsteht damit ein ausgesprochen anticlinischer Bander. Wirbelsäule, wie er anch sonst den Landbewolnern zulsommt.

Die Lenden wirbel (Vertebra lnmbales, Taf. III [XIl], Fig. 5-7) sind leider nur theilweise gut erhalten und es sind deshalb anch die Maasse anf der Tabelle zum Theil nicht ganz präcis, da bei dem meist etwas schief gedrïckten Erhaltungszustand nicht immer ein genaues $\Lambda$ bmessen zu ermöglichen ist.

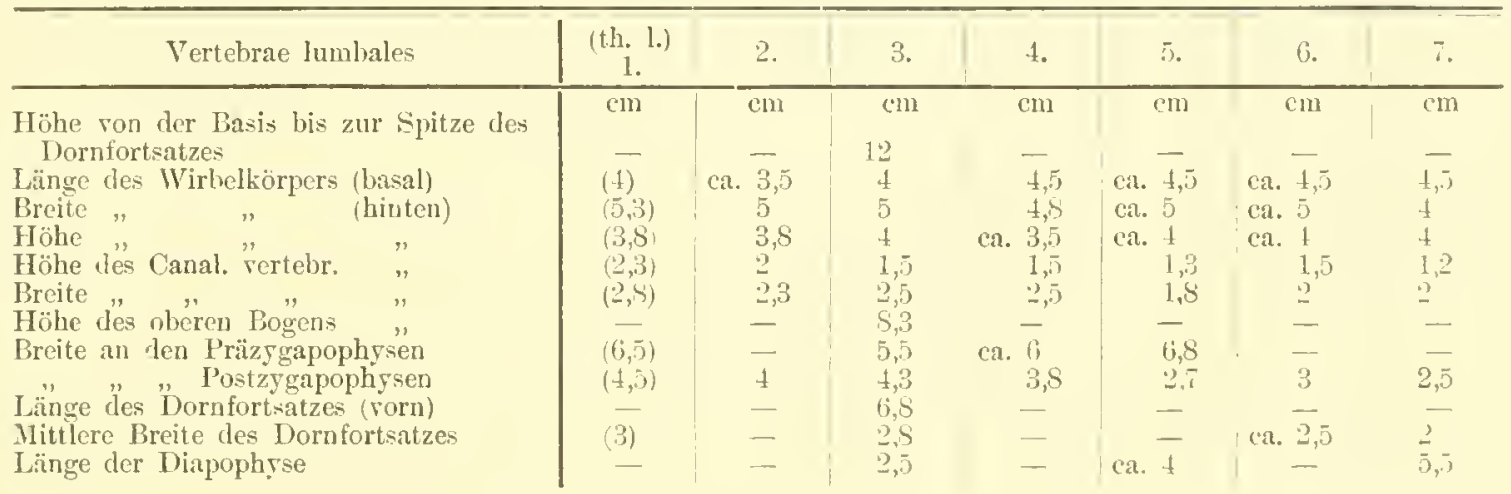

Als Uebergangswirbel kann der linterste aus der Reihe der später gefundenen Wirbel angesprochen werden. da er keine Ansatzstelle für eine Rippe aufweist, aber auch noch in ler Lage der freilich abgebrochenen Diapophyse insofern abweicht, als dieselbe, nach der Bruchtläche zn schliessen, selı weit oben an der Ansatzstelle des Pediculus sass. Die Prï- und Postzygapophysen legen beide noch recht weit aus. Der 2. Lendenwirbel ist schlecht erhalten und lässt nur erkennen, dass die Diapophyse bereits etwas mehr heruntergerückt und wohlausgebildet war. Der wohlerhaltene 3. Lendenwirbel (Taf. III [XII], Fig. 5) zeigt, dass der Dornfortsatz noch sehr kräftig und ganz wenig nach vorne gerichtet war. Die Prä- und Postzygapophlysen sind zwar wollausgehildet, jedoch olıne weitere Fortsätze, wie wir sie als Metapophysen und Anapophysen bei den Landraubthieren kennen. Die Diapophyse ist kurz und steht rechtwinklig vom Wirbelkörper ah. Die 3 nächsten Lendenwirbel ( . lumb. 4, 5 und 6) sind sehr gleichartig gebildet, aber leider in Folge ilıres verdrückten Zustandes nur wenig zur Untersuchung geeignet. Als besonders charakteristisch darf bei diesen das weite Auslegen der Präzygapoplysen gegenïher den kurzen und gedrängt stehenden P'ostzygapophysen Geolog. u. Palännt. Abh., N. F. VI. (der ganzen Feihe X.) Bd., Heft 3. 
betrachtet werden. Die Diapophỵsen nehmen nacl hinten an Stärke und offenbar auch an Länge zu, stehen aber annähernd rechtwinklig vom Wirbelörner ab. Der 7. Lendenwir]el (Taf. III [XII], Fig. 6 u. 7) ist, wieler besser erlalten mul in Folge ler liräftigeren, etwas nach vorne geneigten Diapopliyse selır charakteristisch. Auch bei ihm ist der Dornfortsatz, noch auffallend gut entwickelt und annälıernd senkrecht stehend. Die Form des Wirhelkïrpers weicht von derjenigen der voranstehenden Wirbel insofern ab, als er viel weniger breit erscheint. doch mag dies auch anf den Erhaltungszustand zurïckzufïhıren sein.

Sacralwirbel (Taf. III [XII], Fig. \& nur 9). Es liegt noch ein weiterer Wirbel vor, der leider auch nicht besonders gut erhalten ist, aber doch Interesse verdient, da es sich wohl ohne Zweifel um einen Sacralwirbel handelt. Der Wirhelkörper ist gegeniiber den rorangehenden Lendenwirbelu auffallend niedrig, indem er doppelt so breit als hoch ist, die Unterseite ist ausgeflacht zwischen den tief unten sitzenden Diapophysen. Diese sind sehr breit, gerade abstehend und endigen am distalen Theile mit einer Terdickung, welche kanm anders zu deuten ist als dadurch, dass hier ein Beckenknochen befestigt war. Der obere Bogen ist verschiedenartig von den vorangelenten Wirbeln gebant, indem er an Stelle des medianen Dornfortsatzes 2 in spitzem Winkel zn eimander gestellte Processus obliqnomamillares, aufweist. Ob hier noch Gelenlifacetten ausgebillet waren lässt sich leider in Folge des schlechten Erhaltungszustandes nicht feststellen. In ıer Ausbilung des oberen Bogens trïgt dieser Wirbel bereits den Typus der Schwanzwirbel, wie sie STroner beschieben hat und von welchen leider an unserem Exemplare nichts melı erhalten ist.

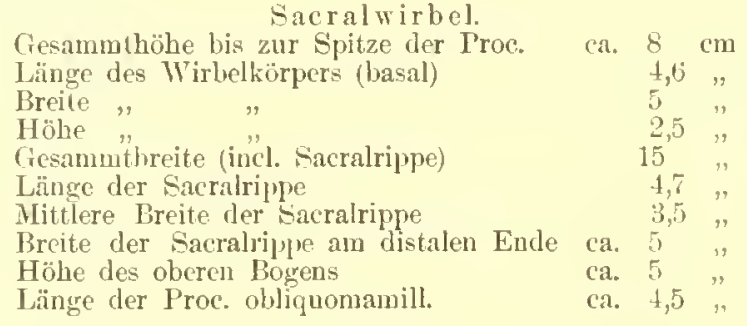

Rippen (Taf. HI [XII], Fig. 1, 10-12): Leider konnte bei der Präparation auf die Rippen am wenigsten Rïcksicht genommen werder, zulem da dieselben meist verdrïckt und versehoben im Gestein lagen und sich nicht herausschälten. So kommt es, dass mir ansser einem Haufwerk von kleinen Fragnenten nur 6 proximale Endigungen vorliegen. Bei allen Rippen fïllt auf, dass sie ungewöhnlich schwach und zierlich gebaut sind; die Länge ist leiler nicht bekannt; der Querschnitt ist am Rippenhalse ein ovaler, wird ajer alluählich rundlich im weiteren Verlanfe der Rippe. Das proximale Ende ist ansgesprochen zweiliöpfig, wie wir schon aus der Beschaffenheit der Wirbel schliessen konnten. Das Tuberculum articulirte an der Diapophyse, wihrend das Capitulum intervertebral an oberen Theile des Wirbelkörpers einlenkte. Bei den hintersten Rippen verschwindet das Tuberculnm fast ganz, nnd das Capitulum articulirt bei den letzten 3 Brustwirbeln nicht mehr intervertebral, sondern in eimer Grube zwischen Pediculus und Wirbelkörper.

Leider ist damit nuser Material erschöpft, und es ist hoffentlich der nächsten Zulmuft vorhehalten, die noch vorhandenen Lücken auszufüllen, insbesondere auch noch Kilarheit ïber den Schwanz, das Extremitätenskelet und den Brust- mnd Beckengürtel zu bringen. Immerhin müssen wir aber zugestehen, dass auch so schon ein Material vorliegt, das jerlenfalls zum vollständigsten gehört, was uns von einem Zeuglodonten bekannt ist. 
Ueberblicken wir das Material in seiner Gesammtheit, so fällt uns in erster Linie ein gewisses Missverhältuiss zwischen dem Schädel und Rumpfe anf, denn der Schädel erscheint mus viel zu gross im Verhältniss zu den zierlichen Gliedern der Wirbelsäule. Reihen wir die Wirbel an einander und lassen ansserdem noch zwischen den einzehnen Wirhelkörpern einen Zwischenraum, obgleich dieser bei den wohl ausgebildeten vorleren und hinteren Gelenkflächen nur gering gewesen sein kann, so bekommen wir ungefähr folgende Proportionen:

$\begin{array}{lr}\text { Schädel } & \text { ca. } 0,60 \mathrm{~m} \\ \text { Hals } & " 0,20 \text { " } \\ \text { Brustregion } & " 0,15 \text { " } \\ \text { Lendenregion (+ Sucralwirhel) } & \text { " } 0.35, "\end{array}$

Ich zweifle nun nicht daran, dass der Schwanz ansserordentlich liräftig und lang war, um einigermaassen das Gleichgewicht wiederherzustellen, aber dennoch haben wir es hier mit einer Eigenthümlichkeit zu thum, die um so auffälliger ist, da wir ja sicher ein Seesäugethier vor uns haben, bei welchem wir einen langgestreckten, fischähulichen Körper anzmnehmen gewohnt sind. Noch auffälliger aber wird die Erscheinung, wenn wir die grossen amerikanischen Zenglodonten als Vergleichsmaterial beiziehen. Selien wir auch ganz ab von der widersinnigen A. Koch'schen Reconstrnction seines Hydrarchos, so leluren uns doch die Maassverhältnisse des von J. MüLLer so eingehend hearheiteten Materiales, dass diese Arten ganz rerschiedenartig gestaltet waren. Wir haben zwar leider keine grösseren sicher zusammengehörigen Theile der Wirbelsänle, doch scheint mir J. MÜLLER ganz richtigg die Schädel und Wirbel seiner Zenglodon macrospondylus und brachyspondylus zusammengestellt zu haben. Danach wỉide Z. brachyspondylus bei einer Schädellänge von ca. $0,80 \mathrm{~m}$ eine Länge des mittleren Brustwirbels von 0.14 und des Lendenwirbels ron 0,20 aufweisen, und bei dem grossen $Z$. macrospondylus kömnen wir eine Schälellänge von ca, 1,20 m annehmen, wozu mittlere Brnstwirbel von $0,24 \mathrm{~m}$ und Lendenwirbel von $0,30 \mathrm{~m}$ Länge gerechnet werden dürfen. Während also im Vergleich zu unserer Art die Schälel von Z. bruchyspomlylus nur $1 / 3$, von Z. macrospondylus doppelt so gross waren, stellt sich das Verhältuiss der mittleren Brustwirbel wie $1: 4$ resp. 1:7, das der Lendenwirbel wie 1:4,4 resp. 1:8. Der Rumpf bis zum Becken war demnach im Verhältniss zum Schädel bei $Z$. Jrachyspondylus 3mal, bei Z. macrospondylus gar 3,5-4nal grösser als bei unserer Art, ja wir dürfen wohl sagen. das s sich das Verhältniss Zw ischen Schädel und Pumpf gerade gedreht hat, denn während bei unserer Art der Schäılel unverhältnismässig gross erscheint. ist er bei den amerikanischen Arten klein zu nennen. ganz besontlers leei Z. muriospontylus.

Sind schon diese Unterschiede in den Proportionen sehr anffällig, so werlen wir noch mehr ïberraseht durch den grmudverschiedenen Bau der einzelnen Wirhel. Bei den grossen Zenglodonten lässt sich ein gewisser Anklang an die Walthiere nicht verleugnen, obgleich die hohen Dornfortsätze an den Brustwirheln und das Abfallen derselben gegen die Lendenregion einen nicht unerheblichen Unterschied bilden, während anderseits der Bat der Lenden- und Schwanzwirbel viel Uebereinstimmendes zeigt. Bei unserer Art fällt der Cetaceencluakterin der Wirbelsäule ganz weg, und wenn wir einen Anschlnss und Vergleich suchen, so werden wij unbedingt zu den Raubthieren greifeu müssen, hei welchen wir alle die Charaktere wiederfinden, die wir hei unserer Form als leitend angeführt haben. Eine Aenderung und Abweichung finden wil eigentlich nur bei den Halswirbeln, welche gegenïber denen der Rauhthiere verkïrzt erscheinen, was mit der Rückbildung des gesammten Halses bei allen Meeresbewohnern im Zusammenhang steht. In der Ausbildung tler Fortsätze jedoch künnen wir aber auch bej den Ualswirbeln leicht den Bau der Raulthierwirbel wiedererkennen. 
So werden wir elenso wie hei den vergleichend-anatomischen Studien des Schädels auch bei denen der Wirhelsäule auf denselheu Wey gewiesen und kommen deshalh mit Sicherheit zu dem Schlusse, dass unsere Art einen Anklang an die alten Raubthiere verrïl und von diesen abgeleitet werden muss. Mit den Creokluntiern stimmt nun anch an besten das anfïillige Missverhältniss zwischen schü̈lelgrösse und Rumpflänge überein, denn soweit uns lis jetzt ganze Slielete ron Creodontiem, wie Hyamontom,

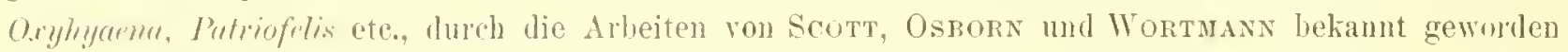
sind, fällt uns stets die Grösse des Schädels im Verhältniss zum Rumple auf. Freilich tritt dies bei unserer Art boch in verstïktem Naasse auf, aber wir müssen uns daran crimern, dass die Länge des Schäclels in Wesentlichen auf die secundïre Streckung des Schnauzentheiles zurückzuführen ist, und dass ohne diese der Schäldel in seinen Verhïlnissen zum Rumpfe gegenüber den Creodontiern nichts Aussergewöhnliches zeigen würle.

Vergleichen wir unsere Art mit den übrigen uns bekannten Zenglodonten, so bietet einerseits der Schälel, abgesehen von der Bezahnung, eine fast vollständige Uebereinstimmung, sowohl mit den amerikanischen Arten, wie mit Zenglodon Osinis, anderseits zeigt aber die Wirbelsänle eine so vollständige Abweichung, dass wir dieselbe ohne Kemntniss des Schädels wohl nie mit den echten Zenglodonten in eine firupe stellen wïrlen. Dies veranlasst mich, für unsere Form anch ein nenes (ienus anfustellen, das ieh Protoeptus als den ersten beliannten Meersänger neme. und da alle unterscheidenden Merkmale sich als primitive Anklänge an die landlebende Gruppe der Creodontier herausstellten, so nenne jeh unsere durchaus nene Art Protoectus atarus.

Aus dem Materiale, das Stroner gesammelt und beschrieben lat, lernen wir in Zinglodon Zitteli eine Art kennen, die zweitellos sich an Protocetus atarus anschliesst und mindestens im Ban der Wirbelsäule mit unserer Art so wahe rerwandt ist, dass sie generisch damit vereinigt werden unss. Die wenigen Wirlel, welche ŚTrouer lueschrielien, stimmen mit Protoctus atrus nahezu vollständig überein, indem sie sich gleichfalls von allen echten Zenytodon-Wirbehn durch ihre geringe Giösse und die Ansbildung als echte Landraulthierwirbel unterscheiden. Gegenüber Protoctns aturns sind die Wirbel grösser, insbesondere die Wirbelkörper gestrecliter und solider, die Dornfortsätze hreiter. Ans Protocetus Zitteli bekommen wir anch Aufsehluss über die Schwanzwirhel, die liräftig gebant, mit breiten Querfortsätzen unck wie wir os bereits beim Sacralwirhel kennen gelernt haben, mit Processus obliquomamillares anstatt der Dornfortsätze verselien simb. Ansserdem lernen wir aus Profoctus Zitteli den wohlentwickelten und complicirten Nasengang liennen, den STroner eingehend beschreibt mud der gleichtalls in vieler Minsicht an die Landthiere erinnert.

Wir kömnen unsere Beohachtungen in folgender Diagnose zusammenfassen:

riotoectus n. $\%$.

I'ntoretus ist dem Ban seines Schädels nach ein rilied der Archänceti oder Urwale, unterscheidet sich aber von den Zeuglodmtiden durch die Ausbildung des Gebisses und des Rumpfes. Das Gebiss ist vollzällig mit : Incisivi. I Canin, 4 Prämolaren, 3 Holaren im Oberkiefer; Unterkiefer nicht bekannt, aber wohl ebenso bezahnt. Die Backenzälne sind nicht zenglodont als Zackenzähne ausgebihlet, sondern lassen sich als secodonte Zähne mit rückgebildeten Innenhöckern auffassen, dementsprechend sind auch die hinteren Zälnne dreiwnrzelig. Die Molaren stehen anf der Innenseite des Jugalfortsatzes der Maxillaria ziemlieh frei, da der harte (iamen nicht bis an die Molaren heranreicht. Die Choanengrube wie bei den Zenglonlonten weit nach hinten gerïckt, vordere Gaumendurchbrïrhe nicht hekannt; die Nascngrube weiter vorne als bei Zenglorlon. dochbogen kräftig, die Orbita klein, unten offen, oben 
von dem kräftigen Processus postorhitalis begrenzt. Die Crista in einen weit vorspringenden. dachfömigen Fortsatz ansgezogen. Die Tympanica als grosse Bullae entwickelt, aber mit dem Schädel fest verwachsen, dahinter grosse Periotica. Der Schädel im Verhältniss zum Rumpfe sehr gross. Der Hals verkürzt, sämmtliche Wirbel zicrlich, som Bau der Landraubthiere, mit lohen Dornfortsåtzen, die eine Anticline bilten: Sacralwirbel wollentwiclielt, zur Betestigung eines Beckens geeignet; rom Sacralwirbel an rückwärts treten Processus obliquomamillares an Stelle der Dornfortsïtze. Die Rippen schlank und zweiköpfig. Brust und Peckengïrtel, sowie die Extremitäten unbeliannt.

Vertretel :

1) Protocotus "ta Kleine Art, mit einer Schädellänge von ca. 0,60 m bei einer Länge les Rumpfes bis zum Sacralwirbel von nur $1 \mathrm{~m}$. Primitivste his jetzt belannte Form mit Charakteren, die sich durchgehend auf die Creodontier, speciell auf die Irorimerrac (Sculosser) beziehen lassen. Bildet den Typus für Protocetus.

2) Protocetus Zittoli Stromer, aus dem oberen Mitteleocän des Fajûm.

Syn. Zneglorlon Zitteli Stoner (Beiträge z. Paläont. unl Geol. Oesterreich-Ungarns und des Orients. Bd. 15. 1903. pag. 82\%.

Soweit bekannt, im Wesentlichen mit Protocetus atnus ibereinstimmend. Etwas grösser und kräftiger gehant mit hochentwickeltem Nasengang.

\section{Mesocetus Schueinfurthi E. FRAAs.}

1883. Srn. Zeuglodon species major Daves. Sitzungsber. d. Kgl. Preuss. Akad. d. W., Phys.-math. Cl. Bd. 6. pag. 129.

1903. "Zeuglodon sp. 11. Stromer, Beitrïge z. Geol. u. Paläont. Oesterreich-Lngarns n. des Orients. Bd. 15. pag. S3.

Wie bemerkt wurde das ron Stroner beschriebene Material eines grossen Zenglodonten durch nene Funde, insbesondere durch den eines Schädels vermehrt, so dass die Art festgestellt werden kann.

Dej Schädel (Taf. I [X], Fig. 3) ist leider nicht gut erhalten und erwies sich als stark rerdrïckt, doch konnte er ans tenn harten Gesteine frei heranspräparirt werlen und bildet auf diese Weise ein recht ansebnliches Stiirk, das uns immerhin über einzelne Slielettheile Aufschluss giebt.

Die Maassverhältnisse, soweit messbar, ergeben Folgendes:

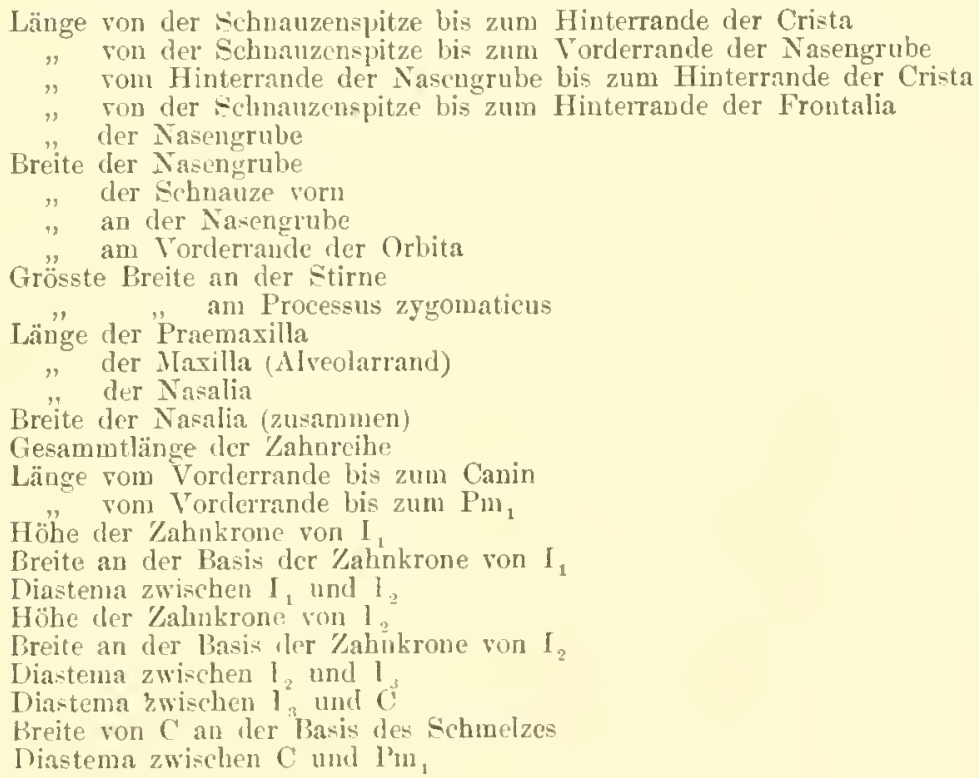


Fïr die Anatomie des Schädelbanes bietet nnser Fremplar wenig Neues, indem die Terhältnisse sich ganz an die beliannten Arten auschliessen. Bezüglich der Grösse liommt es etwa dem Schärlel des Haarlemer Mnsenms (Zenglodon brachyspomlylus .J. MüLLER) gleich und steht benlentend hinter den grossen Exemplaren ron Z. murnospomlylus zurück. Dagegen ist die Schnauze kräftiger gebant, rerjüngt sich aber nicht wie bei $Z$. Osiris, somdern gleicht in dieser Hinsicht dem I'rotoretus aturus. Der Vorderranu der Nasengrube schneidet mit dem Ifintemande des Canins ab und ist wie bei den amerikanischen Arten wohlbegrenzt, zum Thuterschiede von Z. Osiris, wo die Nasengrube nach vorn in einer Pinne rerläuft. In Folge rer zurickliegenden Nasengrube sind die Nasalia rerhälnissmässig kurz. Die Frontalia legen mit ihrem Processus postorhitalis weit aus und iiberdachen die kleine Augenhöhle, die jedoch zu sehr unter dem Gebirgsdluck Noth gelitten hat, um genane Messungen zu erlauben. Interessant ist das wohlausgebildete Jugale, das eine ‘,7 cm hreite, nach hinten zugespitzte Konochenspange bildet und offenbar mit dem weit vorgreifenden Processus zygomaticus des Squamosum in Verbindung tritt. Hinter den Stirnbeinen ist del Schädel ausserordentlich stark eingeschniirt und bildet eine scharfe Crista, deren hinteres Ende aber leider nicht erhalten ist, ebenso wie die ganze Ilinterhan jtsregion.

Die Zähne sind nur im vorderen Schumzentheile gnt erhalten. Die Incisiven bilden liäftige, nach rnickwärts gekrïmmte Ǩegelzähne mit starkem, fein gerumzeltem Schmelz, der eine leichte Kiante erkennen lässt. $I_{2}$ ist noch stärker als $I_{1} ; I_{3}$ war beiderseits ausgefallen. Der Canin war gleichfalls als kräftiger liegelzahn wie die Incisiven entwickelt. Tom Pms ist nur noch die Wurzel sichtlsar, welche bei $t$ cm Länge und 1,1 cm breite anf einen kleineren mol Hacheren Kegelzahn als $C$ hinweist. Die noch erhaltenen Fragmente von Prämolaren lassen erkemen, daß diese eine kräftige Hanptspitze mul wahrscheinlich nur je eine vordere Nebenspitze ansgebihlet hatten und dass der Schmelz auf der Innenseite viel weiter an Zahn herabreichte als auf ler Aussenseite. Es waren ke ine e cll te $n$ Z a cke $\mathrm{z}$ ähne wie bei len ïhrigen bekannten Zenglodonten, sondern sie zeigen Verhältuisse, wie wir sie in ansgezeichneter Weise bei Protortus atacks kennen gelernt haben. Leider simu die Uelor'reste zn dürftig, um ein sicheres Trtheil über die gesammte Bezahnung zu gewinnen, loch liisst sich wenigstens einiges feststellen. So deuten die Fragmente von $\mathrm{Pm}_{2}$ daranf hin. dass derselbe mindestens 5,5 cm lang, also aussergewöhnlich gross war; die Reste ron 2 Wurzeln und einem wohlansgebildeten hintelen Höcker sind erhalten. Tom Pm (Taf. II [XI], Fig. 10) ist llie hintere Ilälfte erhalten; auch an diesem erkennen wix einen kräftigen, hinteren Höcker, welcher sich an die mitlere Ilauptspitze aulegt, deren Schmelz auf der Innenseite sich weit an der Zahnwnrzel herabzieht: der Zahn war zweiwnzelig. Ein linker $\mathrm{II}_{3}$ (Taf. II [XI], Fig. 11) wurde zwar erst s]äter gefunden, gelı̈rt aber offenbar zu demselben Exemplare. Die erhaltene Zahnkrone ist verhältnissmässig klein. $2.8 \mathrm{~cm}$ lang, an der Basis 1,5 cm dick, nnd anf der Innenseite schon angekant, worans wir anf einen entsprechenden $\mathbf{M}_{3}$ im Unterkiefer schliessen dïrlen. Anch bei diesem $M_{3}$ rermissen wir den für die sonstigen Zenglodonten charakteristischen Ban als Zackenzahn, indem er nur eine weit rorragende Hauntkrone und cine lileine hintere Nebenspitze anfweist. An Vorderande ist. del Schmelz verilickt und abgeplattet, wodurch eine Art von rndimentärer Nebenspitze entsteht. Median verlänft eine scharfe liante, auf welcher ler Schmelz sägeförmig gefaltet ist. Anf ler Aussenseite greift der Schmelz nur 2,1 cm, auf der Innenseite dagegen 3,5 cm lierab, wodurch sich ein einseitiger Pau ergiebt, der vollständig mit dem S. 11 hesprochenen Ban des $\mathbf{M}_{3} \mathbf{v o n}$ Protocetus atarus ïlereinstimmt.

Wir sehen demnach, dass unser Zenglodonte im Zahubau durchans verschieden von allen echten Zeuglodonten ist, und dass er sich hierin vollkommen an Frotocetus atarms aureiht. 
Cranz im Gegensatz zu Protoctus finden wir num aber den Isan des Rumpfes.

Die Wirbel simd vou Daues und besonders eingehend ron E. STromer beschrieben worden, so dass ich mich der Anfgabe enthoben fühle, nochmats darauf einzugehen. zudem da das neu beigebrachte Material nichts Nenes bietet. Es möge nur hervorgehoben sein, dass die Wirbel ausserordentlich gross sind nud Verhältnisse bieten, wie wir sie von dem grossen amerikanischen Zeuglodon macrospondylus kenmen. Hier ist lemnach, im Gegensatz zu Protocetus, der Sehädel klein im Verhältniss zum Rumpfe, und die Wirbel haben alle Charaktere der Landranbthiere verloren und die der Cetaceen angenommen.

Somit bietet unsere Art eine interessante Zwischenform zwischen Protocetus und Zcuglodon, indem sie im Ban des Schädels nnd Rumpfes bereits echten Zeuglodontencharakter trägt, im Gebiss dagegen noch den alten P'rotoctus-Typus hat. Dies soll anch der Name Mesoretns ansdrücken, indem ich unsere Art als einen Vorläufer der echten Zenglodonten anffasse, in welchem die Umformung des Körpers bereits vollendet ist, wogegen das Gebiss noeh den primitiven Charakter trïgt. Ich labe diese Art dem verdienstrollen Forscher Aegyptens, Professor Dr. G. Schwetnfurth, dem wir ja anch die ersten Reste dieser Art verdanken, als Zeichen meiner V'erehrung gewidmet.

Es ergiebt sich folgende Diagnose, die hoffentlich bald durch neue Funde erweitert wird:

Mesocetus Schueinfurthi E. FraAs, anssergewöhnlich grosse Art ans der Grenze vom unteren und oberen Mitteleocän ron A gypten. Bildet ein ausgesprochenes Zwischeuglied zwischen Protocetus und Zcuglodon. Gehört dem Bau des Rumpfes nach in die Gruppe von Znuglodon macrospondylus, mit auffallend grossen, langgestreckten Wirbeln von Cetaceencharakter. Der Sclädel im Verhältniss zu den Wirbeln klein, aber immerhin grösser als bei $Z$. Osiris, im Uebrigen vollkommen den übrigen Zenglolonten sich anschliessend. Die Schnanze kräftig, nach vorne kamm zugespitzt, die Nasengrube zuriickliegend, auch nach vorne abgegrenzt, Crista anffallend scharf und

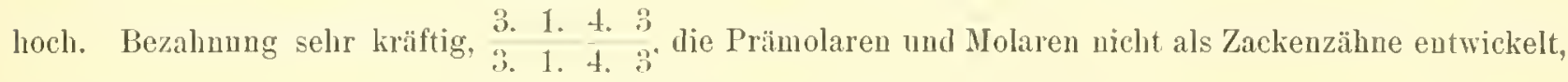
sondern vom Typus secodonter Zähne mit rüickgebildetem Innenhöcker.

So sehen wir, dass wir in dem Eocän yon A e gy pten nicht nur eine grössere Anzahl von Zeuglodonten haben. sondern dass dieselben anch unter sich eine der interessantesten Entwickelnngsreihen darstellen, die unsere Kenntniss über die Stammesgeschichte der Archïoceten erweitern. Den Ausgangspunkt bildet der anch geologisch älteste Protocetus atarus, der im oberen Mitteleocän noch durch Protocetus Zitteli vertreten ist. Von ilm führt _lesocetus Scluceinfurthi über zu den echten Zeuglodonten, welche durch Zeuglodon Osiris vertreten sind.

Zum Schlusse möchte ich noch anf die systematische und phylogenetische Bede utung unserer nenen Funde hinweisen. Dass die Protoceti und Mesoceti zusammen in die Gruppe der Archaeoceti gehören und sich an die Zeuglodontidae anschliessen, unterliegt wohl keinem Zweifel. Bei allen finden wir einen vollständig analogen Schädelbau und, wenn ich mich so ausdruicken darf, dieselbe Tendenz in der Entwickehng. Diese können wir auffassen als die Umwandlung eines Landthieres in einen Meeresbewohner. Die Anpassung an das Wasserleben ist bei den Protoceti zwar anch schon weit vorgeschritten, aber doch nicht so meit, dass wir nicht den ursprünglichen Bau der Landform gewissermaassen durchschimmeru sehen. Bei den Zenglodontiden ist sie so weit vorgeschritten, dass sotrohl in der Bezahnung, ganz besonders aber im Ban der Wirbelsänle die alten Charaktere vollkommen verwiselit und nene aufgetreten sind, die sich aus der Anpassung an das nene Element ergeben. Im Ban des Schädels aher ist noch sehr woht der alte Raubthierschälel zu erkennen. der in vollem 
Gegensatz zu dem der Denticeten und Mysticeten steht und mit liesen rigentlich nur die Streckung ler Schnanze gemein luat, Alles, was wir an den Archaenceti als Anliang an dic Wale mnd Pinnipedier sehen, lässt sich vergleichend-anatomisch nur sehr gezwungen für dic Entwickelungsgeschichte derselben verwenden, was sich schon laraus ergieht. dass wir hahl Anschluss an die Denticeten und Mrsticeten. bald an die Pinnipedier hekommen, obgleich diese heilen (irupren plylogenetisch doch ganz gewiss nichts mit einander gemein haben. Viel klarer und rerständlicher wird has Bilı, wenn wil diese Anklänge an die Neersänger als eine Cunvergenzerscheinung auffassen. hervorgerufen durch die Anpassung an das Wasserleben. Es sind rliesclben Principien und Naturgesetze, nach denen sich in frỉheren Perioden auch einzelne Gruppen iler Reptilien durch Anjassung an las Meerleben umgeformt liaben, und welche sowohl den Ichthyosanriern wie den Thalattosuchiern, Mosasauriden, Dolichosauriern und anderen einen einheitlichen Typus aufprïgen. Ich habe diese Grumbrincipien der Umformung in meiner Arbeit über die Thalattosuchier ${ }^{1)}$ eingehend besprochen. und F. Nopcss ${ }^{2}$ ) hat weitere interessante Bolege dafür beigehracht. Es wurle darauf hingewiesen, dass bei allen diesen Formen unter Zugrumdelegung des primitiven Slieletes einer Urlandform mehr mul mehr die körperform des Fisches angestrebt wird und in diesem Sinne die Umwandhung vor sich geht.

Unter diesem Gesichtspunkte möchte ich auch die Gruppe der Archaeoceti aufgefasst wissen. Die Protoceti geben nns Klarheit ïher die Abstammung derselben von len Creodontiern unn treten als vermittelnule Grupe zwischen die Creolontier und die Zeuglodontidae. Weiterhin ist aber ller Stamm nicht zu verfolgen, nenn die Tebereinstimmung mit den Squalodontiden und damit len Denticeten ist nur eine scheinbare und lässt sich weller in Schädelban noch im übrigen Skelet nachweisen. Noch gezwungener erscheint ein Anschluss an die Mysticeten und gar an die Piminedier. Diese sind jeweils wieder selbständige Grupren, die unter sich wohlbegrenzt sind, deren Abstammung wir aber zur Zeit nicht kennen. Bei allen treten gewisse Merkmale als convergente Erscheinungen auf, die leicht zu dem Irrthum führen, sie in verwandtschaftliche Beziehungen zu bringen.

Systematisch betrachtet trenne ich die lrchacoceti vollständig von den Cetaceen nud schliesse sie als Untergruple an die Creodontieran, während die übrigen Walthiere nach wie vor eine selbstandige Gruppe bilden, die so lange beliehig in der Systematik eingoschattet werden kann, his wir deren Stammesgeschichte kennen. Dass auch die Stammformen der Pinnipedier unter den Creodontiern zu suchen sind, lıalte ich nach den diesbezüglichen Untersuchungen von VTORTMANN $^{3}$ ) für mehr als wahrscheinlich und daraus erlibiren sich anch die vielfachen Uebereinstimmungen zwischen den Archacoceten und Pinpipedieru hesonders im Schädelbau. Figentlich verwandschaftliche Reziehungen können wir aber daraus kaum construiren, denn die Fntwickelung schlng bei heiden ganz gesonderte Wege ein, und rollständig ausgeschlossen ist der Gedauke, lass etwa die Zeuglodonten sich wieder zu halben Landhieren und Künstenhewolnern, wie es lie Pimmipedier larstellen, rückgchildet haben. Immerhin sind die verwandtschaftlichen Beziehungen zwischen den Archäoceten und Pinnepediern insofern genähert, als sie einem gemeinsanen Urstamm von Landthieren, nämlich den der Creorlontier, entsprossen sind.

1) E. FRaAs, Die Meercocodilier (Thalattosuchia) des oberen Jura etc. Palaeontograph. Bd. 49. 1902. pag. $3 \mathrm{ff}$.

2) F. V. Nopcsa, Ueber die Varanusartigen Lacerten 1striens. Beitr. z. Paläont. u. Geol. Oesterreich-I'ngarns u. des Orients. Bd. 15. 1903. Heft 1.

3) J. L. Wortmaxx, Osteology of Patriofelis etc. Bull, of. the American Museum of Nat. Hist. Vol. 6. 1894. Article 5. pag. 157.

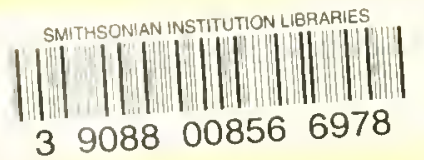



